Published in Chemosphere 90 (2013) 28-35

http://dx.doi.org/10.1016/j.chemosphere.2012.07.002

(C) 2012 Elsevier Inc. All rights reserved

\title{
Comprehensive mass flow analysis of Swedish sludge contaminants
}

\author{
Ulrika Olofsson ${ }^{\mathrm{a}}$, Eva Brorström-Lundén ${ }^{\mathrm{b}}$, Henrik Kylin $^{\mathrm{c}}$, Peter Haglund, a
}

Department of Chemistry, Umeå University, SE-901 87 Umeå, Sweden, IVL Swedish Environmental

Research Institute, P.O. Box 21060, SE-100 31 Stockholm, and Norwegian Institute for Air Research, P.O. Box 100, NO-2027 Kjeller, Norway

*Corresponding author phone: +46 90 7866667; e-mail: peter.haglund@chem.umu.se.

a Umeå University.

${ }^{\mathrm{b}} \mathrm{IVL}$ Swedish Environmental Research Institute.

${ }^{c}$ Norwegian Institute for Air Research.

\section{Abstract}

A screening of metals, persistent organic pollutants, pharmaceuticals and personal care products (PPCPs), and other organic contaminants in sludge from seven Swedish sewage treatment plants (STPs) was performed in this study. This extensive screening provides information on mass flows of 282 compounds used in the Swedish society to sewage sludge. It reveals constant relative contaminant concentrations ( $\mathrm{ng}-\mathrm{mg} \mathrm{kg}^{-1} \mathrm{~d}$.w.), except for some pesticides and perfluorinated compounds, indicating that these originate from broad usage and diffuse dispersion rather than (industrial) point sources. There was a five order of magnitude difference in the sum concentrations of the most and least abundant species (metals and polychlorinated dibenzo-p-dioxins and -furans, respectively). Lower total concentrations were found in sludge from STPs processing primarily food industry or household sewage. Proportions of the amounts used (in Sweden) found in sludge were lower for compounds that are present in consumer goods or are diffusely dispersed into the environment (0.01-1\% recovered in sludge) than for compounds used as detergents or PPCPs (17$63 \%$ ). In some cases, the recovery seemed to be affected by evaporation (e.g. octamethylcyclotetrasiloxane) or biotransformation (e.g. adipates) losses, while polychlorinated alkanes and brominated diphenyl ethers were recovered to disproportionately high degree (ca.4\%); likely due to incomplete statistics for imported goods.

Keywords: PPCP, POP, sewage sludge, emerging pollutants.

Highlights:

- We have performed an extensive screening of sewage sludge contaminants.

- Overall levels and distribution patterns of the contaminants were quite similar between the sewage treatment plants (STPs).

- Levels of sludge contaminants seem to be independent on location, size and treatment technique of the plants.

- Types of human activities connected to STPs, generally, did not affect the levels of sludge contaminants. 


\section{Introduction}

Municipal sewage treatment plants (STPs) process sewage from a variety of sources, e.g. households, industrial sites, and hospitals, and the final solid product is usually dewatered digested sludge. Sludge contains nutrients and organic matter that should be used in agriculture as soil improvement, but also substances that pose environmental and health hazards. The fate of chemicals during sewage treatment depends on both the treatment process and the nature of the substance; by volatilization, biodegradation, sorption to sludge or discharging into recipient water (Zitomer and Speece, 1993). STPs can be considered as secondary sources of pollutants discharged into the environment (Harrison et al., 2006; Kinney et al., 2006; Xia et al., 2005).

Large quantities of pharmaceuticals and personal care products (PPCPs) may enter the aquatic environment via STPs and due to their negative effects on aquatic organisms (DeLorenzo and Fleming, 2008; Kuriyama et al., 2005; Muirhead et al., 2006) PPCPs are classified as emerging pollutants. Domingo and Bocio (2007) concluded that frequently high consumption of certain fish species could increase the health risks by polychlorinated dibenzo- $p$-dioxins and -furans (PCDD/Fs) and polychlorinated biphenyls (PCBs) intake. Results from intake studies (Darnerud et al., 2006; Kiviranta et al., 2004; Schecter et al., 2001) concluded that levels of persistent organic pollutants (POPs) in fish are generally higher compared to other foods. Darnerud et al. (2006) also showed that fish (major), meat, dairy products, and fats/oils are sources to POPs intake for Swedish consumers. Harrison et al. (2006) concluded that more data are needed on contaminant concentrations in sludge as well as temporal trends to enhance our knowledge about their presence in sludge. This is especially of importance during risk assessment of land applications of sludge and to identify needs (if any) for further restrictions. As phosphorus resources will be depleted (Steen, 1998), land applications of biosolids (treated sewage sludge) are important in terms of sustainable nutrient management. Clarke and Smith (2011) concluded that emerging organic compounds (OCs) will not put human health at risk when recycling biosolids on land.

In Sweden, the annual production of STP sludge amounts to approximately 240000 tonnes dry weight (d.w.) per year (Swedish EPA, 2007), with phosphorus and nitrogen contents of ca. $3 \%$ and $3.5 \%$, respectively. Optimizing the use of these nutrients in the sludge could be highly significant in terms of conserving resources. According to a Swedish Government decision, by 2015 at least $60 \%$ of the phosphorus originating from sewage should be returned to productive soil, half of which should be applied to arable land (Swedish EPA, 2002; Wallgren, 2001). The strategy for meeting this objective includes: (i) increasing the recovery of nutrients and (ii) reducing the contents of hazardous substances. The greatest challenge may be to reduce the contents of hazardous substances in sludge and sludge-amended soil sufficiently to fulfill legal requirements and to convince consumers and consumer organizations that products grown on such amended soil are safe.

The purposes of this study were to: (i) perform a screening of metals, POPs, PPCPs, and other OCs in sludge from Swedish STPs with various characteristics; (ii) investigate possible correlations between the annual quantities used in Sweden and the measured concentrations in sewage sludge; (iii) assess whether the percentages found in sludge (relative to the amounts used) are related to physicochemical properties or biodegradability of the pollutants; (iv) examine distribution patterns between and within STPs; and (v) identify potential sources of considered pollutants (e.g. domestic, industrial or storm water). 


\section{Material and methods}

\subsection{Selection of compounds and STPs}

The target compounds and the STPs were selected together with the Swedish EPA, additional information can be found in supplementary data. Main application areas for the compounds and annual quantities used in Sweden (2004) are summarized in Table 1, and physicochemical properties in Table 2 and S1. Table 3 and Fig.S1 presents descriptive data of the STPs.

\subsection{Analysis and data evaluation}

The analyses were performed by several qualified laboratories, each following strict quality guidelines. Generally, internal standard quantification was used to compensate for losses during clean-up and analysis. For non-accredited analyses the extraction efficiencies were checked (e.g. using re-extraction) and found to be sufficient (better than 95\%). The uncertainties of the analytical methods were in the range $20-40 \%$ (for detailed information on the analytical techniques applied see Table S2).

Mass flows (MFs) of the compounds were calculated, based on the measured concentration (MC) and the annual production (in Sweden) of sewage sludge, assuming that all sludge generated is spread on land (that is a reasonable approximation, since only small proportions are incinerated). To assess the proportions of the substances used in the technosphere that reach the STPS and associate with sludge, MFs were compared to the recorded amounts used in Sweden (Swedish Chemicals Agency, 2007b).

Principal component analysis (PCA), a multivariate data analysis tool, was used (SIMCA-P+11; Umetrics, Sweden) to extract and visualize major patterns and trends in the data and to generate an easily interpreted overview of the results. The PCA model includes the compounds that have been analyzed in all STPs $(n=7)$ and results $<$ LoD (limit-of-detection) were set to $1 / 2$ LoD. The data were mean centered and scaled to unit variance to make all parameters equally important.

\section{Results and discussion}

\subsection{Sludge quality}

The total and relative concentrations of the target compounds (Table 4 and S3) were found to be comparatively constant on d.w. basis, indicating that sludge contaminants originate from broad usage and diffuse dispersion rather than from (industrial) point sources, even though industries also contribute to chemical constituents in the sludge. Lower levels of the pollutants (35\%, on average) were detected in sludge from STPs $C$ and G, processing large quantities of water from a food industry (rich in organic substances and lean in pollutants) and household sewage (lower in industrial pollutants), respectively, than in sludge from the other STPs.

Currently there is European maximum residue limits (MRLs) established for seven metals (in Sweden: $\mathrm{Cd}, 2$; $\mathrm{Cr}, 100 ; \mathrm{Cu}, 600 ; \mathrm{Hg}, 2.5 ; \mathrm{Ni}, 50 ; \mathrm{Pb}, 100$; and $\mathrm{Zn}, 800 \mathrm{mg} \mathrm{kg}^{-1}$ d.w.) in sludge for agricultural purposes; there are no legal limits for OCs. However, recommended limit values (Sweden) have been set for three groups of "indicator" OCs, i.e. PAHs ( $3 \mathrm{mg} \mathrm{kg}^{-1} \mathrm{~d} . w$.), PCBs (0.4 mg $\mathrm{kg}^{-1}$ d.w.), and 4-nonylphenol (50 $\mathrm{mg} \mathrm{kg}^{-1} \mathrm{~d} . w$.) (Swedish EPA et al., 1995). All pollutants measured in 
this study were below their respective limit. This also applies to the suggested limits in the EC "Working document on sludge" (CEC, 2000) (Di-(2-ethylhexyl) phthalate, DEHP, 100; 4-nonylphenol, 50; $\sum \mathrm{PAH}_{9}, 6$; and $\sum \mathrm{PCB}_{7} 0.8 \mathrm{mg} \mathrm{kg}^{-1}$ d.w.; $P C D D / F s, 100 \mathrm{ng}$ TEQ kg ${ }^{-1}$ d.w.).

In order to obtain an overview of the sludge contaminants, currently not regulated, the median of the total concentration of each group of compounds detected at each STP was used (Fig.1). There was a five order of magnitude difference in the total concentrations; with metals detected in highest and PCDD/Fs in lowest concentrations. Currently, the EC is assessing if the more than 20-year old sludge directive should be reviewed and revised (http://ec.europa.eu/environment/waste/sludge/index.htm). The data generated in this and other studies provide a wider database of sludge contaminants and aid in the discussions of future limit values.

\subsection{Use pattern and sludge contaminant mass flows}

The intended use of consumer chemicals, the principal emission routes from articles in use in society (molecular or particulate) and long-range air transport (reaching the sewer system via storm water systems) will all affect the sludge quality. In the case of emissions from consumer articles it is expected that molecular emissions are (relatively) more important for volatile compounds and particulate emissions more important for semi-volatile (high molecular weight) compounds. Comparisons of quantities used in the Swedish society and annual MFs for sludge (Table 4) may be utilized to identify use related, STP treatment related, and other factors affecting the sludge quality.

Metals are used in large quantities in the society and this is reflected in the MF-value (190 tonnes year $^{-1}$ ) which is highest of all compound classes. Of the remaining abundant compound classes (with MFs $>250 \mathrm{~kg}$ year $^{-1}$ ) a majority originates from PPCPs (siloxanes, triclosan (antibacterial), BHT (antioxidant), and fluoroquinolone (FQ) and tetracycline (TC) antibiotics), detergents (4-nonylphenol) or pesticides (incl. biocides); products that are used in such a way that release to the sewer system is inevitable. Many of these compounds (siloxanes, 4-nonylphenol, FQs, TCs, and triclosan) have high affinity to sludge (Buyuksonmez and Sekeroglu, 2005; IVL, 2005; Lindberg et al., 2006; Ying and Kookana, 2007). Consequently, it is logical that high proportions of the used amounts are recovered in sludge. Of the annual quantities used in Sweden, 63\% of the FQs, $51 \%$ of the TCs, $41 \%$ of 4-nonylphenol, $29 \%$ of triclosan, and $17 \%$ of the siloxanes were found sorbed to sludge.

High MFs (>10 tonnes year ${ }^{1}$ ) were also obtained for two groups of high volume plastic additives (Table 4), phthalates (plasticizers) and polychlorinated alkanes (PCAs; flame retardants and plasticizers). These pollutants are presumably evaporating at a low rate from plastic materials, partitioning to dust, and reach the sewage system following cleaning of indoor environments. Although the emission rate is expected to be low, the stock of plastic materials in society is large and the resulting total emitted amounts substantial.

The percentages of PCAs, and the closely related polybrominated diphenylether flame retardants (PBDEs), found sorbed on sludge were remarkably high (4.1 and 3.7\%, respectively) - much higher than for organophosphate triesters (OPs; plasticizers and flame retardants) and phthalates (0.06 and $0.04 \%$, respectively). Since all of these classes of compounds have low volatility and, thus, presumably low emission rates, the 10-fold higher percentages recovered of PCAs and PBDEs are hard to explain by intrinsic physicochemical properties. Instead, it may be due to limitations of the national use statistics, not including chemicals in imported articles. It is likely that considerable 
amounts of PCAs and PBDEs are entering the country as (unrecorded) additives in imported textiles, plastic articles, computers, and other electronic goods, etc.

Many of the compounds that were found at relatively low concentrations in sludge (average concentration $<1 \mathrm{mg} \mathrm{kg}^{-1}$ d.w.; MFs $\leq 0.15$ tonnes), e.g. polycyclic aromatic hydrocarbons (PAHs), organotin compounds (OTCs), terpene, chlorophenols (CPs), PCBs, polychlorobenzenes (PCBz), perfluorochemicals (PFCs) and PCDD/Fs stem from multiple, often diffuse sources, such as traffic and long-range air transport, that can reach STPs via storm water systems. For these chemicals it is difficult to make meaningful comparisons with use statistics.

\subsection{Factors affecting mass flows}

It should be noted that many other factors, in addition to loads of pollutants at STPs, affect their levels in the sludge. The pollutants may, for instance, evaporate/degrade during the treatment process, or pass the STP due to highly water solubility and low sludge affinity. These processes are often related to the physicochemical characteristics (Table 2 and S1) and biodegradability of the compounds.

\subsubsection{Evaporation}

The volatility of the contaminants may affect the MF-values in two ways; it may (i) affect the initial emission rates of additives in consumer articles and (ii) affect the evaporative losses in the sewage treatment process. A linear relation was observed between the molecular weights of plastic additives and the percentage recovered in sludge $\left(R^{2}=0.94\right.$; Fig.2), which may indicate that the latter process is more important than the first (for these compounds). However, the compounds included in Fig. 2 have such low vapor pressure (Henry's law constants $<10^{-5} \mathrm{~atm} \mathrm{~m}^{3} \mathrm{~mol}^{-1}$ ) that substantial evaporation is unlikely. These compounds are therefore most likely emitted from consumer articles mainly through particulate emissions or leaching upon washing or cleaning (OPs; water solubility's $>5 \mathrm{mg} \mathrm{L}^{-1}$; Table S1).

Evidence for considerable evaporative losses in STPs was however found for cyclic methylsiloxanes. The proportion of their quantities used that reach STPs and sorb to sludge seems to strongly depend on their vapor pressures, which decrease with the number of siloxane units. Octa- (D4), deca- (D5), and dodeca-methylcyclotetrasiloxane (D6) were recovered at $1.3 \%, 17 \%$, and $54 \%$, respectively. The losses may occur either during biological sewage treatment or sludge digestion. The latter process is known to result in tainted biogas that cause deposits in boilers fed such fuel (Dewil et al., 2006, 2007). Similarly, volatilization is probably the reason for the relatively low recovery of limonene (0.46\%).

\subsubsection{Biodegradation}

Unlike the other PPCPs, the non-steroid anti-inflammatory substances (NSAIDs) were recovered to a low degree $(0.04 \%)$ in sludge, which may indicate biodegradation and/or passage through STPs in aqueous phase. The latter explanation is less likely as antibiotics are more water soluble and recovered to a greater extent (about 50\%). Moderate (diclofenac; 32\%) to high (ibuprofen, naproxene, and ketoprofen; 65-90\%) removal efficiencies has been reported for Swedish STPs (IVL, 2006). The cited study also showed that the removal efficiency varies considerably between STPs, 
and that the removal efficiency tends to be lower in STPs located in the northern part of Sweden, possibly due to lower water temperatures (and lower biological activity).

The percentage recovered of long-chain phthalates (Fig.2) seems to correlate to their molecular weight, maybe due to less availability for biodegradation of the larger molecules. Similarly, of the eight adipates investigated, only the largest (di-(2-ethylhexyl) adipate, DEHA) was detected in sludge. Earlier studies have reported that most of the phthalates are biodegradable in sludge (Liang, et al. 2008).

Biodegradation may also be responsible for the low percentage $(0.01 \%)$ of tributyl phosphate (TBP) recovered in sludge (Fig.2). TBP has lower water solubility than tris(2-chloropropyl) phosphate (TCPP) and should therefore associate more strongly to sludge, but are still recovered to a 10 -fold lower degree. From reports in the scientific literature it is known that TBP and other aliphatic OPs are more degradable than aromatic OPs (e.g. triphenyl phosphate, TPP) and that chlorinated OPs (e.g. TCPP) are most persistent (Saeger et al., 1979; WHO, 1991a, 1991b, 1998).

\subsubsection{Water solubility}

The proportions of OPs found in the sludge $(0.06 \%)$ were much lower than PCAs and PBDEs (Table 4) although they are used in similar applications. This may be partially due to recent substitution of the latter by OPs that will immediately influence the statistics, but only slowly affect the levels in sludge (due to long lifetime and large stocks in use). However, the OPs generally have higher biodegradability (3.3.2.) and higher water solubility than PBDEs. Hence, larger proportions of these substances may be degraded or pass through STPs. Chlorinated OPs such as tris(2-chloroethyl) phosphate (TCEP) and TPP are both persistence and water soluble and have been shown to pass STPs to a great extent (Marklund et al., 2005).

\subsection{Detection of point sources and differences in use pattern}

The PCA model was generated to obtain an overview of the relationships between individual pollutant concentrations (or groups of pollutants) and individual STPs (or groups of STPs). Points representing all STPs, except STP E, cluster near the origin of the score plot (Fig.3a), indicating that these plants have similar pollutant profiles. STPS A, B, D, and F are separated to some degree from STPs $C$ and $G$, generating sludge with lower overall pollutant levels than the other STPs, hence confirm earlier statement (3.1.).

The PCA loading plots show that sludge from STP E has higher proportions of most PFCs (Fig. $3 \mathrm{~b}$ and Fig.S2) - various pesticides (Fig.3c), hexachlorobenzene (HCBz), highly chlorinated PCDD/Fs (Fig.3d), 4-nonylphenol (4NP) and PBDE 209 (Fig.3e) than the other STPs. The high levels of PFCs in its sludge may be related to textile factories and associated facilities that are connected to STP E and may use these substances during their manufacturing processes or, alternatively, the compounds may be present in imported materials they use. The relatively high levels of the highly chlorinated PCDD/Fs may be related to use of pentachlorophenol as fungicide, e.g. during storage/transport of fabrics, consistent with previous analyses of various municipal solid waste fractions that have shown that the textile fraction contains highest percentages of total PCDD/Fs (Hedman et al., 2007). It may be worth noting that the Swedish textile industry no longer uses perfluoroalkylsulfonates (PFOS, PFDS, PFHxS), and that the STP handling sewage from the textile industry (STP E) contains similar concentrations of PFOS as the other STPs. In fact, sludge from the two largest STPs (A, B) and STP D 
contains more perfluoroalkylsulfonates than STP E. Imported products that contain PFCs (excluded in the product register) are assumed to be the primary current sources of PFCs in Swedish STPs.

Sludge from STPs A, B, and D also contains large proportions of metals, antibacterial agents (triclosan and OTCs), and two phthalates (DEHP and di-iso-nonyl phthalate, DINP) that are used as plasticizers in polyvinyl chloride (PVC). These cities are growing rapidly, as their traffic intensity, which may explain the high metal and phthalate levels. The elevated levels of zinc and lead may stem from brake linings of motor vehicles (Hjortenkrans et al., 2007) and metal plated roofs, and the phthalates from building materials. The detection of DEHP at relatively high levels ( $\mathrm{mg} \mathrm{kg}^{-1} \mathrm{~d} . \mathrm{w}$.), although it has mainly been substituted by DINP since early 2000 (Petersson, 2004; Swedish Chemicals Agency, 2007a), indicates that there may be a considerable time lag between regulatory actions and a corresponding drop in pollutant levels, due to the large amounts of DEHP remaining in the technosphere. In one case, STP D, industrial production of vinyl flooring was identified as a possible contributor. Furthermore, all three cities have relatively young and dynamic populations that are likely to buy/use more sports/functional clothing (often containing PFCs/antibacterial agents) than the populations connected to the other STPS.

Sludge produced by the two STPs $(C, G)$ with lowest sludge contaminant levels has relatively high proportions of the fungicide propiconazole, which is primarily applied to barley crops. These STPS are situated in agricultural areas and STP C processes waste from a major food-processing plant, which may explain the occurrence of propiconazole in its sludge.

\section{Conclusion}

The overall levels and distribution patterns of the sludge contaminants are quite similar, hence these parameters do not seem to be dependent on either the location/size/treatment technique of the plants, or generally the types of human activities that affect the waste streams they handle. Using PCA, minor variations in pollutant levels and patterns were identified and attributed to potential sources. There were clear differences in the PFC distribution patterns between the STPs that could be related to textile industries around STPE, and the occurrence of relatively high levels of propiconazole in sludge from STPS $C$ and $\mathrm{G}$ could be associated with agricultural activities.

The proportions of the amounts used found in sludge were generally lower for compounds that are incorporated in consumer goods than for compounds used as detergents or PPCPs and pollutants emitted from point sources. Some (weak) correlations were found between the national use statistics of the chemicals and their measured concentrations in sludge. However, the product registry does not include chemicals in imported goods. Even for articles produced in Sweden it is difficult to determine how much of the various chemicals actually are emitted from the products and reach the sewage system. Thus, there is a need for generic reliable emission models for chemicals in consumer articles.

\section{Acknowledgements}

We gratefully acknowledge the financial support from the Environmental Monitoring Program of the Swedish EPA. Ulf Järnberg (ITM, Stockholm University), Solomon Tesfalidet (Umeå University), and Mikael Remberger and Lennart Kaj (IVL, Stockholm) are thanked for performing chemical analysis, and the STP personnel for help with sampling. 


\section{Supplementary data}

Additional information regarding the selection of the OCs, sample collection, analysis and QA/QC, location of the STPs, distribution patterns of PFCs, CAS numbers, physicochemical properties, analytical techniques applied, uncertainties in the analyses, and sludge concentrations are presented in supplementary data.

\section{References}

Buyuksonmez, F., Sekeroglu, S., 2005. Presence of pharmaceuticals and personal care products (PPCPs) in biosolids and their degradation during composting. J. Res. Sci. Technol. 2(1), 31-40.

CEC, 2000. Working Document on Sludge, 3rd Draft. Council of the European Community, Brussels 27 April, pp. 19.

Clarke, B. O., Smith, S. R., 2011. Review of 'emerging' organic contaminants in biosolids and assessment of international research priorities for the agricultural use of biosolids. Environ. Int. 37(1), 226-247.

Darnerud, P. O., Atuma, S., Aune, M., Bjerselius, R., Glynn, A., Grawe, K. P., Becker, W., 2006. Dietary intake estimations of organohalogen contaminants (dioxins, PCB, PBDE and chlorinated pesticides, e.g. DDT) based on Swedish market basket data. Food Chem. Toxicol. 44(9), 15971606.

DeLorenzo, M. E., Fleming, J., 2008. Individual and mixture effects of selected pharmaceuticals and personal care products on the marine phytoplankton species Dunaliella tertiolecta. Arch. Environ. Contam. Toxicol. 54(2), 203-210.

Dewil, R., AppelS, L., BaeyenS, J., 2006. Energy use of biogas hampered by the presence of siloxanes. Energy Convers. Manage. 47(13-14), 1711-1722.

Dewil, R., AppelS, L., BaeyenS, J., Buczynska, A., Vaeck, L., 2007. The analysis of volatile siloxanes in waste activated sludge. Talanta 74(1), 14-19.

Domingo, J. L., Bocio, A., 2007. Levels of PCDD/PCDFs and PCBs in edible marine species and human intake: A literature review. Environ. Int. 33(3), 397-405.

EPI Suite ${ }^{\mathrm{TM}}$, 2007. The Estimation Program Interface (EPI) Suite. (C) 2000 U. S. Environmental Protection Agency.

Harrison, E. Z., Oakes, S. R., Hysell, M., Hay, A., 2006. Organic chemicals in sewage sludges. Sci.Tot. Environ. 367(2-3), 481-497.

Hedman, B., Burvall, J., Nilsson, C., Marklund, S., 2007. PCDD/F in source-sorted waste fractions and emissions from their co-combustion with reed canary-grass. Waste Manage. 27(11), 1580-1592.

Hjortenkrans, D. S. T., Bergback, B. G., Haggerud, A. V., 2007. Metal emissions from brake linings and tires: Case studies of Stockholm, Sweden 1995/1998 and 2005. Environ. Sci. Technol. 41(15), 5224-5230.

IVL, 2005. Results from the Swedish National Screening Programme 2004. Subreport 4: Siloxanes. IVL Swedish Environmental Research Institute Ltd., IVL Report B1643.

IVL, 2006. Results from the Swedish National Screening Programme 2005. Subreport 1: Antibiotics, Anti-inflammatory substances, and Hormones. IVL Swedish Environmental Research Institute Ltd., IVL Report B1689. 
Kinney, C. A., Furlong, E. T., Zaugg, S. D., Burkhardt, M. R., Werner, S. L., Cahill, J. D., Jorgensen, G. R., 2006. Survey of organic wastewater contaminants in biosolids destined for land application. Environ. Sci. Technol. 40(23), 7207-7215.

Kiviranta, H., Ovaskainen, M. A. L., Vartiainen, T., 2004. Market basket study on dietary intake of PCDD/Fs, PCBs, and PBDEs in Finland. Environ. Int. 30(7), 923-932.

Kuriyama, S. N., Talsness, C. E., Grote, K., Chahoud, I., 2005. Developmental exposure to low-dose PBDE-99: Effects on male fertility and neurobehavior in rat offspring. Environ. Health Perspectives $113(2), 149-154$

Liang, D. W., Zhang, T., Fang, H. H. P., He, J. Z., 2008. Phthalates biodegradation in the environment. App. Microbiol. Biotechnol. 80(2), 183-198.

Lindberg, R. H., Wennberg, P., Johansson, M. I., Tysklind, M., Andersson, B. A. V., 2005. Screening of human antibiotic substances and determination of weekly mass flows in five sewage treatment plants in Sweden. Environ. Sci. Technol. 39(10), 3421-3429.

Lindberg, R. H., Olofsson, U., Rendahl, P., Johansson, M. I., Tysklind, M., Andersson, B. A. V., 2006. Behavior of fluoroquinolones and trimethoprim during mechanical, chemical, and active sludge treatment of sewage water and digestion of sludge. Environ. Sci. Technol. 40(3), 1042-1048.

Marklund, A., Andersson, B., and Haglund, P. (2005). Organophosphorus flame retardants and plasticizers in Swedish sewage treatment plants. Environ. Sci. Technol. 39(19), 7423-7429.

Muirhead, E. K., Skillman, D., Hook, S. E., Schultz, I. R., 2006. Oral exposure of PBDE-47 in fish: Toxicokinetics and reproductive effects in Japanese medaka (Oryzias latipes) and fathead minnows (Pimephales promelas). Environ. Sci. Technol. 40(2), 523-528.

Petersson Lars, 2004. Miljöredovisning 2003 (in Swedish) (Environmental Report 2003). Tarkett AB, Ronneby.

Saeger, V. W., Hicks, O., Kaley, R. G., Michael, P. R., Mieure, J. P., Tucker, E. S., 1979. Environmental Fate of Selected Phosphate-Esters. Environ. Sci. Technol. 13(7), 840-844.

Schecter, A., Cramer, P., Boggess, K., Stanley, J., Papke, O., Olson, J., Silver, A., Schmitz, M., 2001. Intake of dioxins and related compounds from food in the US population. J. Toxicol. Environ. Health-Part A, 63(1), 1-18.

Steen, I., 1998. Phosphorus Recovery - Phosphorus availability in the 21st century Management of a non-renewable resource. Phosphorus \& Potassium,(217).

STPs, 2005. Miljörapporter 2004 (in Swedish). Environmental Reports 2004.

Swedish Chemicals Agency, 2006. Perfluorinated substances and their uses in Sweden, ISSN: 02841185. Swedish Chemicals Agency, Report Nr 7/06.

Swedish Chemicals Agency, 2007a. Statistics. Swedish Chemicals Agency.

Swedish Chemicals Agency, 2007b. Keml-stat. Swedish Chemicals Agency.

Swedish EPA, The Swedish Water \& Wastewater Association (VAV), The Federation of Swedish Farmers (LRF), 1995. Användning av avloppsslam i jordbruket (in Swedish). The Swedish National Environmental Protection agency, Rapport 4418.

Swedish EPA, 2002. Aktionsplan för återföring av fosfor ur avlopp (in Swedish). The Swedish National Environmental Protection agency, Rapport 5214.

Swedish EPA, 2007. Siffror om avloppsslam (in Swedish). The Swedish National Environmental Protection agency, http://www.naturvardsverket.se/sv/Start/Verksamheter-medmiljopaverkan/Avlopp/Avlopsslam/Siffror-om-avloppsslam/.

Takacs-Novak, K., Jozan, M., Hermeczi, I., Szasz, G., 1992. Lipophilicity of antibacterial fluoroquinolones. Int. J. Pharm. 79, 89-96. 
Wallgren, B., 2001. Swedish policy on phosphorus recovery. Paper presented at 2nd International Conference on Recovery of Phosphates from Sewage and Animal Wastes, Holland, 12-13 March 2001.

WHO, 1991a. Tributyl Phosphate. World Health Organization, Environmental Health Criteria(112).

WHO, 1991b. Triphenyl Phosphate. World Health Organization, Environmental Health Criteria(111).

WHO, 1998. Flame Retardants: Tris(chloropropyl) Phosphate and Tris(2-chloroethyl) Phosphate. World Health Organization, Environmental Health Criteria(209).

Xia, K., Bhandari, A., Das, K., Pillar, G., 2005. Occurrence and fate of pharmaceuticals and personal care products (PPCPs) in biosolids. J. Environ. Quality, 34(1), 91-104.

Ying, G.-G., and Kookana, R. S. (2007). Triclosan in wastewaters and biosolids from Australian wastewater treatment plants. Environ. Int. 33(2), 199-205.

Zitomer, D. H., Speece, R. E., 1993. Sequential Environments for Enhanced Biotransformation of Aqueous Contaminants. Environ. Sci. Technol. 27(2), 226-244. 
Table 1 Primarily applications and annual quantity (in Sweden) of the compounds

\begin{tabular}{|c|c|c|c|}
\hline Compounds & $\mathrm{n}^{\mathrm{a}}$ & Applications & $\begin{array}{l}\text { Quantity } \\
\left(10^{3} \mathrm{~kg}_{\text {year }}\right)^{\mathrm{b}}\end{array}$ \\
\hline As, $\mathrm{Cd}, \mathrm{Co}, \mathrm{Cr}, \mathrm{Cu}, \mathrm{Hg}, \mathrm{Ni}, \mathrm{Pb}, \mathrm{V}, \mathrm{Zn}$ & 10 & Metals & 21046 \\
\hline Phthalates & 8 & Softening agent in plastics & 73964 \\
\hline Biocides & 11 & Killing living organisms & 1637 \\
\hline Polycyclic aromatic hydrocarbons (PAHs) & 6 & Products of incomplete combustion & $1203^{c}$ \\
\hline Adipates & 8 & Softening agent in plastics & 1073 \\
\hline Pesticides & 109 & Control pests & 828 \\
\hline Polychlorinated alkanes (PCAs) & 3 & $\begin{array}{l}\text { Lubricants and cutting fluids (metal working } \\
\text { industry), and plasticizers }\end{array}$ & 298 \\
\hline Organophosphorus compounds (OPs) & 6 & $\begin{array}{l}\text { Flame retardants and/or plasticizers in } \\
\text { textiles, plastics, and building materials }\end{array}$ & 286 \\
\hline Butylhydroxytoluene (BHT) & 1 & $\begin{array}{l}\text { Stabilizer in plastics and rubber, } \\
\text { antioxidant (ex. processed food) }\end{array}$ & 254 \\
\hline Organotin compounds (OTCs) & 6 & $\begin{array}{l}\text { In anti-fouling compositions and stabilizer } \\
\text { in polyvinyl chloride (PVC) }\end{array}$ & 240 \\
\hline $\begin{array}{l}\text { Non-steroid anti-inflammatory drugs } \\
\text { (NSAIDs) }\end{array}$ & 4 & Pharmaceuticals & $84^{d}$ \\
\hline Siloxanes & 7 & $\begin{array}{l}\text { Sanitary articles, lubricants and hydraulic } \\
\text { fluids in textiles and skin care products }\end{array}$ & 31 \\
\hline Perfluorochemicals (PFCs) & 13 & $\begin{array}{l}\text { Water, fat or stain repellents for paper, } \\
\text { textiles, carpets, etc. }\end{array}$ & $24^{\mathrm{e}}$ \\
\hline Terpene (Limonene) & 2 & $\begin{array}{l}\text { Flavor and odor additive (hygiene products } \\
\text { and perfumes) }\end{array}$ & 17 \\
\hline Polychlorobenzenes (PCBz) & 11 & Dyestuffs and solvent for pesticides & $8.1^{f}$ \\
\hline 4-Nonylphenol & 1 & Detergent, surfactants (metabolite) & 7.6 \\
\hline Fluoroquinolones (FQs) & 3 & Pharmaceuticals (antibiotics) & $5.1^{\mathrm{g}}$ \\
\hline Polybrominated diphenylethers (PBDEs) & 8 & $\begin{array}{l}\text { Flame retardants in electronics, furniture, } \\
\text { and building materials }\end{array}$ & $3.6^{\mathrm{h}}$ \\
\hline Triclosan & 1 & $\begin{array}{l}\text { Antibacterial in toothpaste and deodorants } \\
\text { (personal care products) }\end{array}$ & 3.1 \\
\hline Tetracyclines (TCs) & 5 & Pharmaceuticals (antibiotics) & $1.3^{\mathrm{d}, \mathrm{i}}$ \\
\hline Hormones & 4 & Pharmaceuticals & $0.07^{d}$ \\
\hline Chlorophenols (CPs) & 19 & Wood and textile preservatives & $\begin{array}{l}\text { Banned } \\
(1978)^{j}\end{array}$ \\
\hline Polychlorinated biphenyls (PCBs) & & Dielectric fluids in transformers and & Banned \\
\hline WHO-PCBS & 12 & capacitors & $(1972)^{j}$ \\
\hline Indicator-PCBs (I-PCBs) & 7 & & \\
\hline $\begin{array}{l}\text { Polychlorinated dibenzo- } p \text {-dioxins and } \\
\text { furans (PCDD/Fs) }\end{array}$ & 17 & Unintentionally formed (by-products) & - \\
\hline
\end{tabular}

${ }^{a} \mathrm{n}$ : number of compounds included in the group. ${ }^{b}$ Annual quantity, in Sweden 2004, recorded in the products and pesticides register (Swedish Chemicals Agency, 2007b). 'Quantity of benzo(a)pyrene. ${ }^{\mathrm{d}}$ The Swedish Medical Products Agency (MPA). ${ }^{\mathrm{e}}$ Swedish Chemical Agency (2006). ${ }^{\mathrm{f}}$ Quantity of 1,2-dichlorobenzene. ${ }^{\mathrm{g}}$ Lindberg et al. (2005). " $Q$ Quantity of PBDE 209. 'demeclocycline and chlorotetracycline not included. In Sweden. 
Table 2 Physicochemical properties of the studied compounds (EPI Suite ${ }^{\mathrm{TM}}$, 2007)

\begin{tabular}{|c|c|c|c|c|c|}
\hline $\begin{array}{l}\text { Class of } \\
\text { compound }\end{array}$ & Sub group & $\begin{array}{l}\mathrm{M}_{\mathrm{w}} \\
\left(\mathrm{g} \mathrm{mol}^{-1}\right)^{\mathrm{a}}\end{array}$ & $\log K_{\text {ow }}{ }^{b}$ & $\begin{array}{l}\mathrm{S}_{\mathrm{w}} \\
\left(\mathrm{mg} \mathrm{L}^{-1}\right)^{\mathrm{c}}\end{array}$ & $\begin{array}{l}\mathrm{H} \\
\left(\mathrm{atm} \cdot \mathrm{m}^{3} \mathrm{~mol}^{-1}\right)^{\mathrm{d}}\end{array}$ \\
\hline Metals & As, $\mathrm{Cd}, \mathrm{Co}, \mathrm{Cr}, \mathrm{Cu}, \mathrm{Hg}, \mathrm{Ni}, \mathrm{Pb}, \mathrm{V}, \mathrm{Zn}$ & $51-207$ & $-0.6-0.7$ & $6 \cdot 10^{4}-4 \cdot 10^{5}$ & $2 \cdot 10^{-2}-0.8$ \\
\hline Esters & $\begin{array}{l}\text { Phthalates } \\
\text { Adipates } \\
\text { OPs }\end{array}$ & $\begin{array}{l}194-447 \\
202-427 \\
266-431\end{array}$ & $\begin{array}{l}1.7-10 \\
2.4-10 \\
1.6-4.7\end{array}$ & $\begin{array}{l}1 \cdot 10^{-5}-8 \cdot 10^{3} \\
4 \cdot 10^{-6}-690 \\
5-6 \cdot 10^{3}\end{array}$ & $\begin{array}{l}4 \cdot 10^{-8}-4 \cdot 10^{-5} \\
2 \cdot 10^{-6}-2 \cdot 10^{-4} \\
1 \cdot 10^{-11}-3 \cdot 10^{-6}\end{array}$ \\
\hline Pesticides & $\begin{array}{l}\text { Biocides } \\
\text { Insecticides } \\
\text { Herbicides } \\
\text { Fungicides }\end{array}$ & $\begin{array}{l}110-362 \\
183-505 \\
111-412 \\
169-403\end{array}$ & $\begin{array}{l}1-4.7 \\
-0.9-7.4 \\
-4.5-5.3 \\
0.9-5.5\end{array}$ & $\begin{array}{l}7 \cdot 10^{-6}-3 \cdot 10^{5} \\
2 \cdot 10^{-3}-1 \cdot 10^{6} \\
5 \cdot 10^{-2}-1 \cdot 10^{6} \\
4 \cdot 10^{-2}-9 \cdot 10^{3}\end{array}$ & $\begin{array}{l}6 \cdot 10^{-12}-5 \cdot 10^{-7} \\
3 \cdot 10^{-12}-4 \cdot 10^{-4} \\
4 \cdot 10^{-19}-2 \cdot 10^{-4} \\
8 \cdot 10^{-14}-3 \cdot 10^{-5}\end{array}$ \\
\hline Hydrocarbons & $\begin{array}{l}\text { PAHs } \\
\text { Terpene (Limonene) }\end{array}$ & $\begin{array}{l}202-276 \\
136\end{array}$ & $\begin{array}{l}4.9-6.7 \\
4.8\end{array}$ & $\begin{array}{l}2 \cdot 10^{-4}-9 \cdot 10^{-2} \\
44\end{array}$ & $\begin{array}{l}1 \cdot 10^{-7}-8 \cdot 10^{-6} \\
0.4\end{array}$ \\
\hline Phenols & $\begin{array}{l}\text { Chlorophenols } \\
\text { Butylhydroxytoluene } \\
\text { Triclosan } \\
\text { 4-Nonylphenol }\end{array}$ & $\begin{array}{l}129-266 \\
220 \\
290 \\
220\end{array}$ & $\begin{array}{l}2.2-4.7 \\
5.0 \\
4.7 \\
5.9\end{array}$ & $\begin{array}{l}45-1 \cdot 10^{4} \\
10 \\
9.3 \\
2.7\end{array}$ & $\begin{array}{l}1 \cdot 10^{-7}-4 \cdot 10^{-7} \\
4 \cdot 10^{-6} \\
5 \cdot 10^{-9} \\
6 \cdot 10^{-6}\end{array}$ \\
\hline Organometals & Organotin compounds & $177-351$ & $0.6-7.4$ & $6 \cdot 10^{-7}-4 \cdot 10^{3}$ & $1 \cdot 10^{-9}-1.5$ \\
\hline Pharmaceuticals & $\begin{array}{l}\text { Fluoroquinolones } \\
\text { Tetracyclines } \\
\text { NSAIDs } \\
\text { Hormones }\end{array}$ & $\begin{array}{l}319-361 \\
444-483 \\
206-296 \\
272-298\end{array}$ & $\begin{array}{l}-0.3-0.3^{\mathrm{e}} \\
-4--0.7 \\
3.0-4.0 \\
2.8-4.1\end{array}$ & $\begin{array}{l}7 \cdot 10^{3}-4 \cdot 10^{4} \\
160-3 \cdot 10^{5} \\
11-260 \\
13-560\end{array}$ & $\begin{array}{l}5 \cdot 10^{-20}-9 \cdot 10^{-19} \\
1 \cdot 10^{-31}-5 \cdot 10^{-24} \\
5 \cdot 10^{-12}-2 \cdot 10^{-7} \\
1 \cdot 10^{-12}-6 \cdot 10^{-10}\end{array}$ \\
\hline Siloxanes & Methylsiloxanes & $162-445$ & $4.8-6.5$ & $1 \cdot 10^{-4}-1.4$ & $9 \cdot 10^{-2}-0.8$ \\
\hline $\begin{array}{l}\text { Fluorinated } \\
\text { compounds }\end{array}$ & Perfluorochemicals & $314-714$ & $2.2-12$ & $6 \cdot 10^{-7}-2$ & $1 \cdot 10^{-9}-2 \cdot 10^{3}$ \\
\hline $\begin{array}{l}\text { Halogenated } \\
\text { compounds }\end{array}$ & $\begin{array}{l}\text { Polychlorobenzenes } \\
\text { PBDEs } \\
\text { I-PCBs } \\
\text { WHO-PCBs } \\
\text { PCDD/Fs } \\
\text { PCAs }\end{array}$ & $\begin{array}{l}147-285 \\
407-959 \\
258-395 \\
292-395 \\
306-460\end{array}$ & $\begin{array}{l}3.3-5.9 \\
5.9-12 \\
5.7-8.3 \\
6.3-8.3 \\
6.3-9.5 \\
\text { Depend o }\end{array}$ & $\begin{array}{l}0.3-100 \\
3 \cdot 10^{-6}-0.3 \\
4 \cdot 10^{-4}-0.1 \\
4 \cdot 10^{-4}-3 \cdot 10^{-2} \\
9 \cdot 10^{-6}-3 \cdot 10^{-2} \\
\text { te degree of ch }\end{array}$ & $\begin{array}{l}9 \cdot 10^{-4}-3 \cdot 10^{-3} \\
1 \cdot 10^{-8}-7 \cdot 10^{-6} \\
5 \cdot 10^{-5}-2 \cdot 10^{-4} \\
5 \cdot 10^{-5}-1 \cdot 10^{-4} \\
1 \cdot 10^{-6}-2 \cdot 10^{-5} \\
\text { nation }\end{array}$ \\
\hline
\end{tabular}

Abbreviations can be found in Table $1 .{ }^{a} \mathrm{M}_{\mathrm{w}}$ : Molecular weight. ${ }^{b} \log \mathrm{K}_{\mathrm{ow}}$ : octanol-water partition coefficient, estimated values. ${ }^{c} \mathrm{~S}_{\mathrm{w}}$ : Water solubility, estimated values. ${ }^{\mathrm{d}} \mathrm{H}$ : Henry's Law Constant, estimated values. ${ }^{\mathrm{e}}$ Experimentally determined value (Takacs-Novak et al., 1992). 
Table 3 Descriptive data of the investigated sewage treatment plants (STPs) (STPs, 2005)

\begin{tabular}{|c|c|c|c|c|c|c|c|}
\hline STP ID & $\begin{array}{l}\text { Stockholm } \\
\text { A }\end{array}$ & $\begin{array}{l}\text { Gothenburg } \\
\text { B }\end{array}$ & $\begin{array}{l}\text { Eslöv } \\
\text { C }\end{array}$ & $\begin{array}{l}\text { Umeå } \\
\text { D }\end{array}$ & $\begin{array}{l}\text { Borås } \\
\text { E }\end{array}$ & $\begin{array}{l}\text { Alingsås } \\
\mathrm{F}\end{array}$ & $\begin{array}{l}\text { Floda } \\
\text { G }\end{array}$ \\
\hline Inhabitants served & 684000 & 621000 & 17500 & 84000 & 79000 & 24000 & 10000 \\
\hline No. of personal equivalents (pe) & 835000 & 772000 & 100000 & 100000 & 98000 & 37000 & 6000 \\
\hline Dimensioning of the STP (pe) & 900000 & 680000 & 330000 & 116000 & 110000 & 60000 & 13000 \\
\hline Type of activity connected ${ }^{a}$ & Ind. (mix) & Ind. (mix) & Ind. (F) & $\mathrm{H}$ & $\mathrm{H} /$ Ind. $(\mathrm{T}, \mathrm{C})$ & Ind. (L) & House \\
\hline Treatment of the sewage ${ }^{b}$ & $M / C / B / D$ & $M / C / B / D$ & $M / B / C / D$ & $M / C / B / D$ & $M / C / B / D$ & $M / C / B / D$ & $\mathrm{M} / \mathrm{C} / \mathrm{B} / \mathrm{S}$ \\
\hline Solid $t_{r}$ (days) $^{c}$ & 15 & 15 & 30 & 18 & 29 & 17 & 18 \\
\hline Raw sewage water $\left(\mathrm{Mm}^{3}\right.$ year $\left.{ }^{-1}\right)$ & 88 & 117 & 4.6 & 12 & 18 & 4.6 & 1.5 \\
\hline Storm water (\%) & 5 & 50 & 43 & 20 & 59 & 38 & 66 \\
\hline Sewage sludge (tonnes d.w. year ${ }^{-1}$ ) & 14300 & 13500 & 1600 & 2300 & 3500 & 770 & 270 \\
\hline Fraction of total production (\%) & 6.0 & 5.6 & 0.67 & 1.0 & 1.5 & 0.32 & 0.11 \\
\hline Sewage sludge d.w. (\%) & 29 & 28 & 21 & 37 & 22 & 23 & 30 \\
\hline
\end{tabular}

${ }^{a}$ Ind., industry; mix, mixture of industrial sewage; F, food; $\mathrm{H}$, hospital; $\mathrm{T}$, textile; $\mathrm{C}$, chemical; L, laundry; and House, household. ${ }^{\mathrm{b}} \mathrm{M}$, mechanical; C, chemical; B, biological treatment (activated sludge); D, digestion (anaerobic); and S, stabilization (aerobic) of the sludge. 'Solid retention time in the digester/aerobic stabilizer. ${ }^{d}$ Percentage of the total annual production of sewage sludge in Sweden (Swedish EPA, 2007). e Percent dry weight of total mass. 
Table 4 Detection frequencies (Det. Freq.), concentration ranges, measured concentrations (MC), mass flows (MF), and percentage of the compounds found (recovered) in the sewage sludge relative the amounts used

\begin{tabular}{|c|c|c|c|c|c|c|c|}
\hline \multirow[t]{2}{*}{ Compounds } & \multirow[t]{2}{*}{$\begin{array}{l}\text { Det. } \\
\text { Freq. }^{\text {a }}\end{array}$} & \multicolumn{4}{|c|}{$\begin{array}{l}\text { Measured concentrations (MC) } \\
\left(\mu \mathrm{kg}^{-1} \mathrm{~d} . \mathrm{w} .\right)\end{array}$} & \multirow[t]{2}{*}{$\begin{array}{l}\text { MF } \\
\left(\text { kg year }^{-1}\right)^{c}\end{array}$} & \multirow[t]{2}{*}{$\begin{array}{l}\text { Rec. } \\
(\%)^{d}\end{array}$} \\
\hline & & $\min$ & $\max$ & average & $\mathrm{RSD}(\%)^{\mathrm{b}}$ & & \\
\hline $\mathrm{As}, \mathrm{Cd}, \mathrm{Co}, \mathrm{Cr}, \mathrm{Cu}, \mathrm{Hg}, \mathrm{Ni}, \mathrm{Pb}, \mathrm{V}, \mathrm{Zn}$ & $7 / 7$ & 500000 & 1100000 & 800000 & 23 & 190000 & 0.91 \\
\hline Phthalates & $7 / 7$ & 69000 & 320000 & 120000 & 57 & 29000 & 0.04 \\
\hline Polychlorinated alkanes (PCAs) & $7 / 7$ & 9900 & 82000 & 50000 & 56 & 12000 & 4.1 \\
\hline Siloxanes & $3 / 3$ & 13000 & 25000 & 22000 & 33 & 5300 & 17 \\
\hline Biocides & $7 / 7$ & 4000 & 62000 & 14000 & 102 & 3400 & 0.21 \\
\hline 4-Nonylphenol & $7 / 7$ & 8000 & 40000 & 13000 & 26 & 3100 & 41 \\
\hline Fluoroquinolones (FQs) & $7 / 7$ & 7000 & 16000 & 13000 & 62 & 3200 & 63 \\
\hline Triclosan & $7 / 7$ & 1800 & 8300 & 3700 & 51 & 890 & 29 \\
\hline Tetracyclines (TCs) & $2 / 2$ & 1400 & 4100 & 2800 & 70 & 660 & 51 \\
\hline Pesticides & $4 / 7$ & 2100 & 10000 & 2100 & 115 & 500 & 0.06 \\
\hline Butylhydroxytoluene (BHT) & $7 / 7$ & 640 & 2400 & 1500 & 49 & 360 & 0.14 \\
\hline Organophosphorus compounds (OPs) & $7 / 7$ & 310 & 2800 & 730 & 78 & 170 & 0.06 \\
\hline Polycyclic aromatic hydrocarbons (PAHs) & $6 / 7$ & 470 & 1700 & 640 & 76 & 150 & 0.01 \\
\hline Polybrominated diphenylethers (PBDEs) & $7 / 7$ & 390 & 870 & 550 & 28 & 130 & 3.7 \\
\hline Organotin compounds (OTCs) & $7 / 7$ & 230 & 480 & 410 & 29 & 99 & 0.04 \\
\hline Terpene (Limonene) & $2 / 2$ & 150 & 500 & 330 & 75 & 78 & 0.46 \\
\hline $\begin{array}{l}\text { Non-steroid anti-inflammatory drugs } \\
\text { (NSAIDs) }\end{array}$ & $2 / 2$ & 62 & 230 & 140 & 81 & 35 & 0.04 \\
\hline Chlorophenols (CPs) & $6 / 7$ & 34 & 130 & 92 & 61 & 22 & - \\
\hline Adipates & $2 / 2$ & 40 & 100 & 70 & 61 & 17 & 0.002 \\
\hline Indicator-PCBs & $7 / 7$ & 23 & 100 & 50 & 67 & 12 & - \\
\hline Polychlorobenzenes & $7 / 7$ & 20 & 330 & 39 & 126 & 9.3 & 0.11 \\
\hline Perfluorochemicals (PFCs) & $7 / 7$ & 13 & 290 & 38 & 128 & 9.2 & 0.04 \\
\hline WHO-PCBS & $7 / 7$ & 3.2 & 11 & 6 & 44 & 1.4 & - \\
\hline Polychlorinated dioxins/furans (PCDD/Fs) & $7 / 7$ & 0.96 & 2.2 & 1.6 & 25 & 0.4 & - \\
\hline Hormones & $0 / 2$ & $<\operatorname{LoD}^{\mathrm{e}}$ & $<$ LoD & $<$ LoD & - & - & - \\
\hline
\end{tabular}

${ }^{a}$ Number of positive samples/number of STPs. ${ }^{b}$ RSD: Relative Standard Deviation. ${ }^{c}$ MF: Mass Flow, based on MC and the annual production of sewage sludge (Swedish EPA, 2007). ${ }^{d}$ Percentage of the compounds recovered in sewage sludge, of

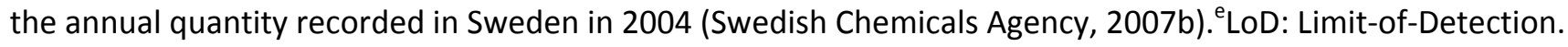




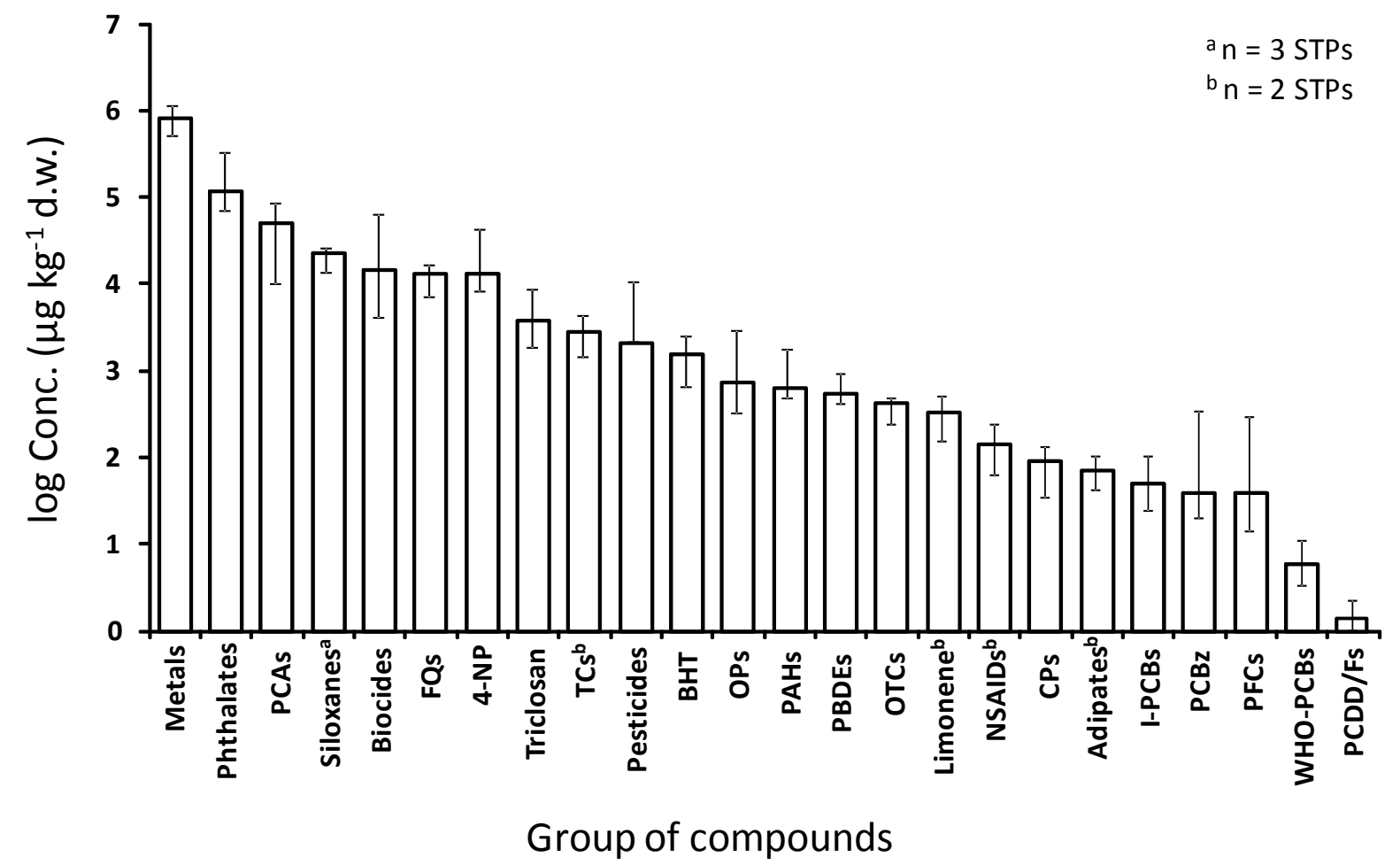

Fig.1. Median total concentrations (logarithmic scale) of each measured group of metals, persistent organic pollutants, pharmaceuticals and personal care products, and other organic contaminants in sludge from sewage treatment plants (STPs) in Sweden. The error bars correspond to the ranges of total concentrations ( $n=7$ STPs, unless otherwise indicated). Abbreviations: PCAs, Polychlorinated alkanes; FQs, Fluoroquinolones; 4-NP, 4-nonylphenol; TCs, Tetracyclines; BHT, Butylhydroxytoluene; OPs, Organophosphorus compounds; PAHs, Polycyclic aromatic hydrocarbons; PBDEs, Polybrominated diphenylethers; OTCs, Organotin compounds; NSAIDs, Non-steroid antiinflammatory drugs; CPs, Chlorophenols; I-PCBs, Indicator-PCBs; PCBz, Polychlorobenzenes; PFCs, Perfluorochemicals; PCDD/Fs, Polychlorinated dibenzo- $p$-dioxins and -furans. 


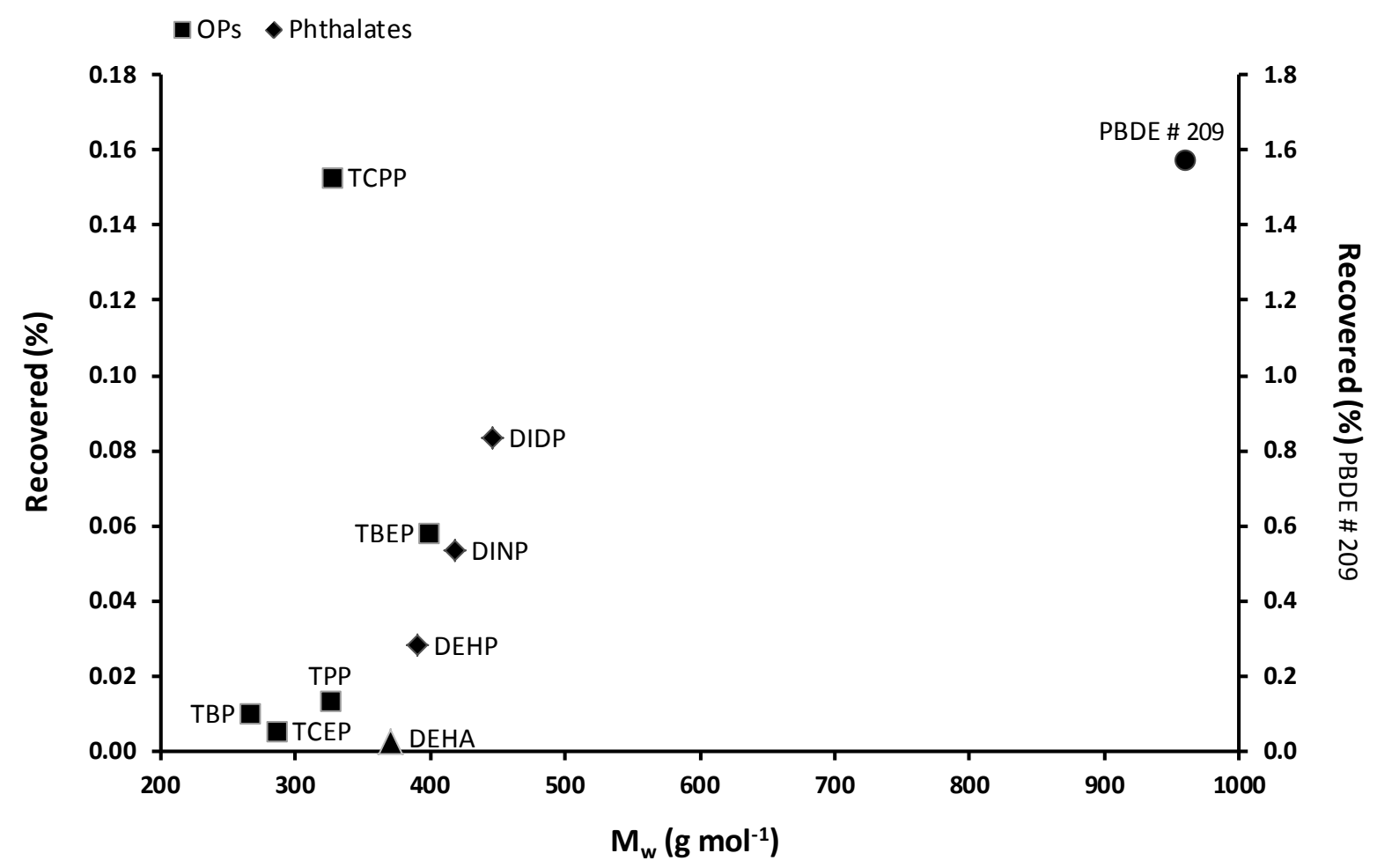

Fig.2. Molecular weight $\left(\mathrm{M}_{\mathrm{w}} \mathrm{g} \mathrm{mol}^{-1}\right)$ vs. percentage recovered (\%) in sludge of plastic additives (plasticizers and/or flame retardants), $\mathrm{R}^{2}=0.94$ (TCPP excluded). OPs, organophosphorus compounds (TBP, tributyl phosphate; TCEP, tris(2-chloroethyl) phosphate; TPP, triphenyl phosphate; TCPP, tris(2chloroisopropyl) phosphate; TDCPP, tris(1,3-dichloro-2-propyl) phosphate); DEHA, di-(2-ethylhexyl) adipate; Phthalates (DEHP, di-(2-ethylhexyl) phthalate; DINP, di-iso-nonyl phthalate; DIDP, di-isodecyl phthalate); PBDE \#209, decabrominated diphenylether. 


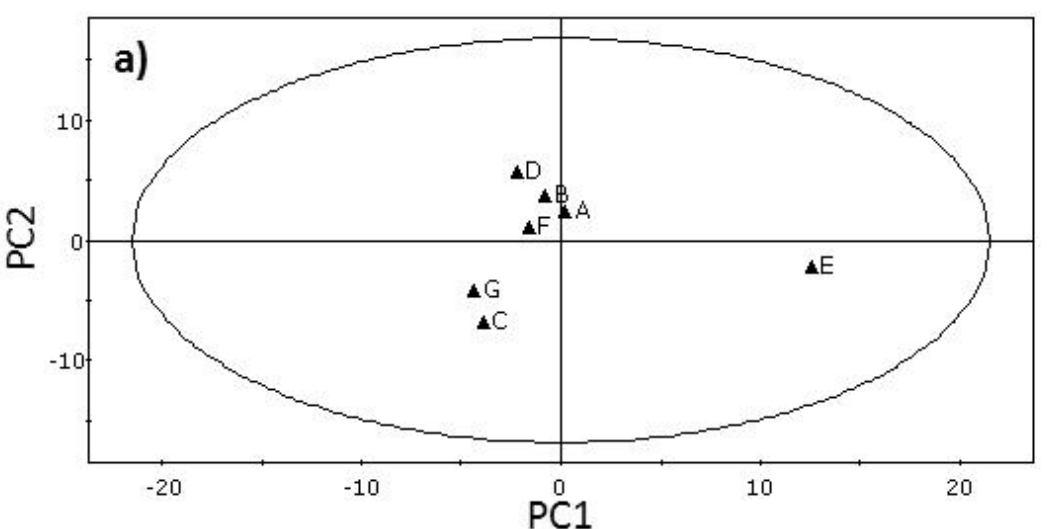

A: Stockholm - large, industry (mix)

$B$ : Gothenburg-large, industry (mix)

C: Eslöv - industry (food)

D: Umeå - hospital

E: Borås - hospital/industry (textile, chemical)

F: Alingsås - industry (laundry)

G: Floda-household
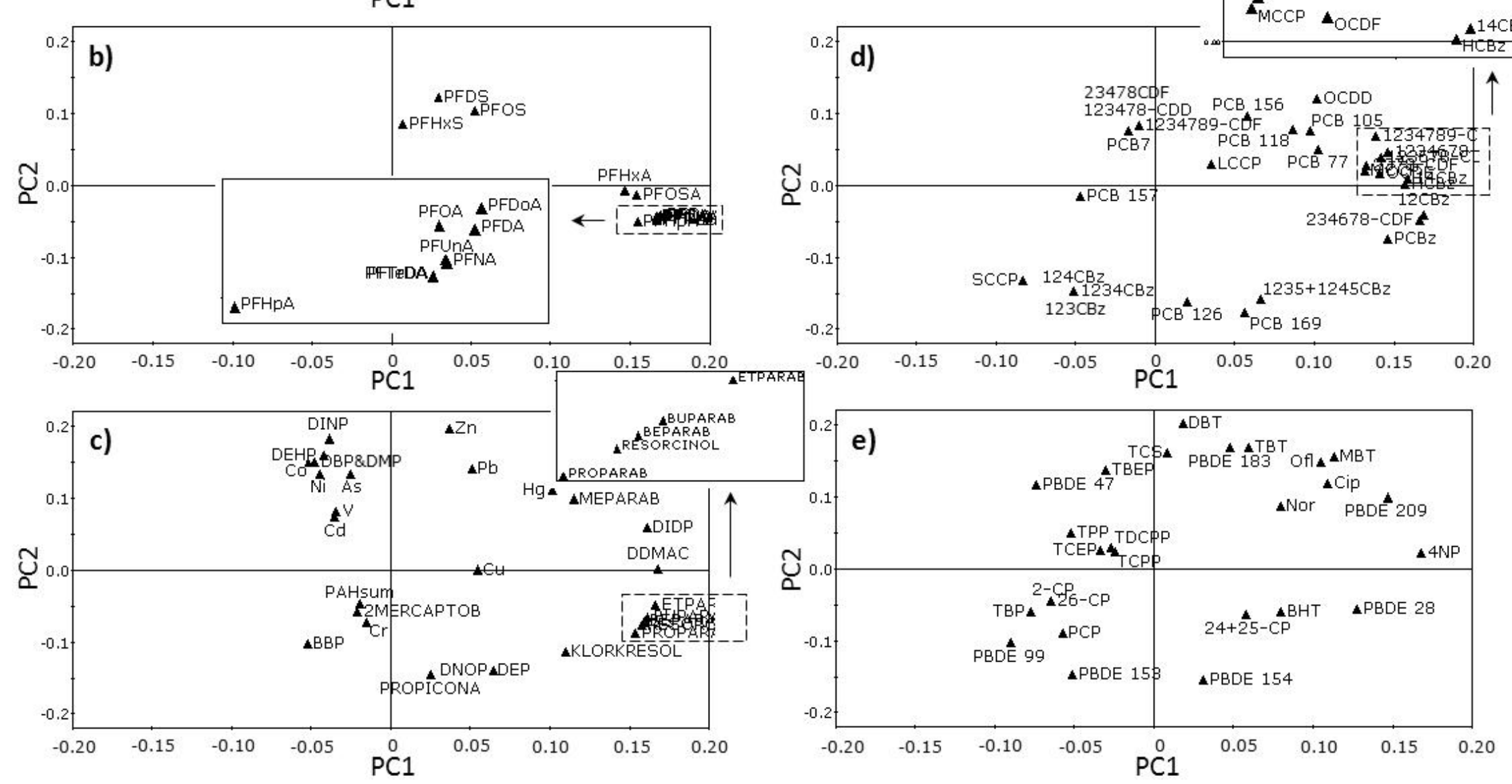

Fig.3. Principal Component Analysis (PCA) plots displaying patterns in levels of contaminants in sewage at the selected sewage treatment plants (STPS), based on measured concentrations of the compounds analyzed in all STPs ( $n=7)$, with levels below the limit-of-detection, LoD, were set to half the LoD. The first and second principal components (PC1 and PC2) explained 34\% and $21 \%$, respectively, of the total variance in the data. a: score plot showing relationships between the Swedish STPs. b-e: loading plots showing the corresponding relationships among the metals, persistent organic pollutants, pharmaceuticals and personal care products, and other organic contaminants. For compound abbreviations, see Table S1. The three congested areas (boxes with broken lines) include: PFDoA, PFOA, PFDA, PFUnA, PFNA, PFTrDA, PFTeDA, PFHpA (panel b); ETPARAB, BUPARAB, BEPARAB, RESORCINOL, PROPARAB (panel c); and 1234789-CDD, 1234678-CDF, 123678-CDD, 2378-CDF, MCCP, OCDF, 14CBz, HCBz (panel d). 
Supplementary data

\section{Comprehensive mass flow analysis of Swedish sludge contaminants}

Ulrika Olofsson ${ }^{a}$, Eva Brorström-Lundén ${ }^{b}$, Henrik Kylin ${ }^{c}$, Peter Haglund*, a

Department of Chemistry, Umeå University, SE-901 87 Umeå, Sweden, IVL Swedish Environmental Research Institute, P.O. Box 21060, SE-100 31 Stockholm, and Norwegian Institute for Air Research, P.O. Box 100, NO-2027 Kjeller, Norway

"Corresponding author phone: +46 90 7866667; e-mail: peter.haglund@chem.umu.se.

${ }^{a}$ Department of Chemistry, Umeå University, Umeå, Sweden.

${ }^{\mathrm{b}}$ IVL Swedish Environmental Research Institute, Stockholm, Sweden.

${ }^{\mathrm{c} N o r w e g i a n}$ Institute for Air Research, Norway.

Pages: 22

Figures: 2

Tables: 3 


\section{Selection of chemicals}

The organic compounds monitored in the Swedish annual sludge sampling program, and included in this study, were selected from Scandinavian priority lists, the European Union Water Framework Directive (WFD) (EU, 2000), or the "Working document on sludge" (CEC, 2000). A wide range of biocides and pesticides are also included (generated from the Swedish EPA emerging pollutants screening program), most of which are restricted in the EU (EU, 1991) and classified as POPs (UNEP, 2001) or as WFD priority substances (EU, 2000). As part of their normal routines the STPs monitor levels of polycyclic aromatic hydrocarbons (PAHs), PCBs, and 4-nonylphenol (compiled from environmental reports).

\section{Sewage treatment plants and sample collection}

Dewatered digested (anaerobic) or stabilized (aerobic) sludge was collected at seven STPs (Table 3 and Fig.S1) spread across Sweden in the autumn of 2004 (unless otherwise stated). These STPS represent large-, medium- and small-sized plants. Samples were collected during periods of normal working and weather conditions. Composite samples $(n=3)$ from each STP were collected in dark bottles within one hour after sludge dewatering. In order to reduce the risk of microbial degradation, the sludge samples were frozen immediately after sampling and stored in freezer at $18^{\circ} \mathrm{C}$ until the chemical analysis.

\section{Analysis and QA/QC}

The chemical analyses were performed at Swedish laboratories experienced in the analysis of the target substances. These laboratories use established laboratory quality assurance and quality control (QA/QC) procedures. In parallel with all samples, method blank samples were run as controls to ensure that any contamination during preparation, clean-up, and instrumental analysis did not significantly influence the quantitative results. For a positive identification the analyte chromatographic retention time had to agree with that of an authentic reference standard and its signal intensity had to be at least $3 \times$ LoD (limit-of-detection). 


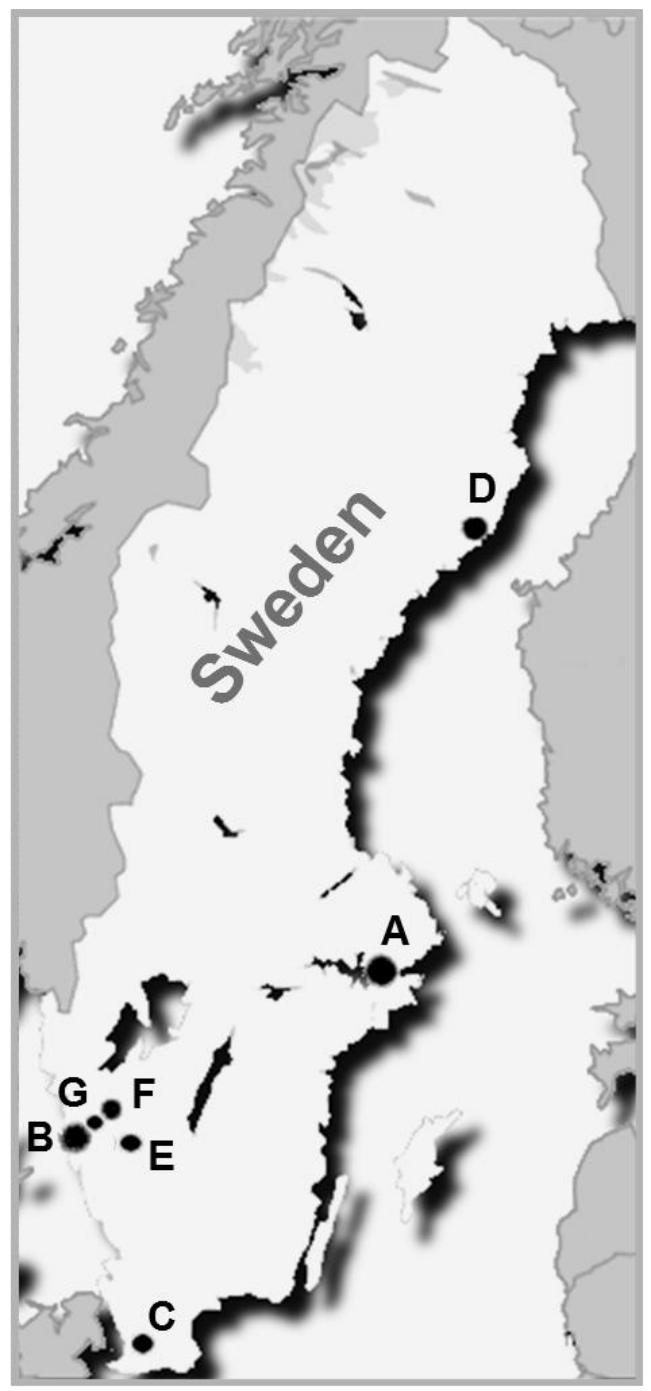

Fig. S1. Map of sampling locations in Sweden, STPs A-G; A, Stockholm; B, Gothenburg; C, Eslöv; D, Umeå; E, Borås; F, Alingsås; G, Floda. 


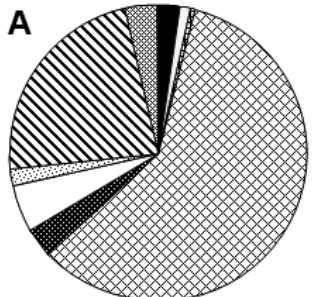

Total: $113 \mu \mathrm{g} \mathrm{kg}^{-1} \mathrm{~d} . \mathrm{w}$ $69 \%$ of PFCs detected

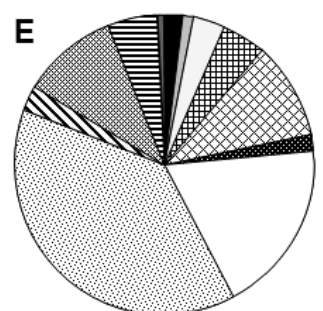

Total: $290 \mu \mathrm{g} \mathrm{kg}^{-1}$ d.w. $100 \%$ of PFCs detected

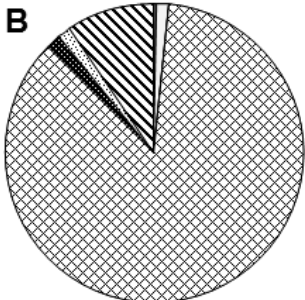

Total: $53 \mu \mathrm{g} \mathrm{kg}^{-1} \mathrm{~d} . \mathrm{w}$
$\mathbf{4 6 \%}$ of PFCs detected

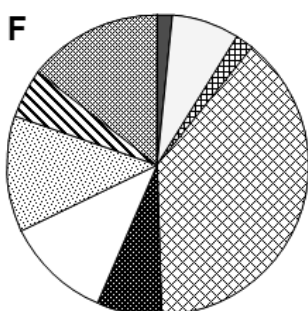

Total: $22 \mu \mathrm{g} \mathrm{kg}{ }^{-1} \mathrm{~d} . \mathrm{w}$ $69 \%$ of PFCs detected

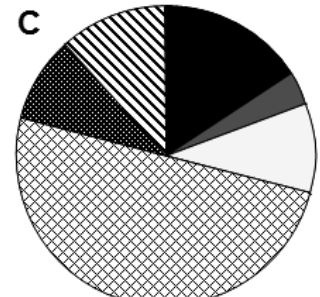

Total: $13 \mu \mathrm{g} \mathrm{kg}^{-1}$ d.w. $46 \%$ of PFCs detected

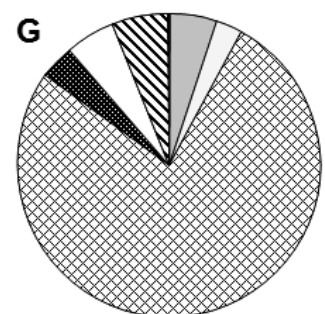

Total: $16 \mu \mathrm{g} \mathrm{kg}^{-1} \mathrm{~d}$.w. $46 \%$ of PFCs detected

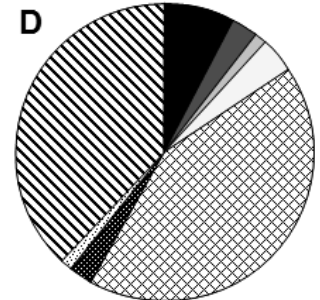

Total: $38 \mu \mathrm{g} \mathrm{kg}^{-1}$ d.w. $62 \%$ of PFCs detected

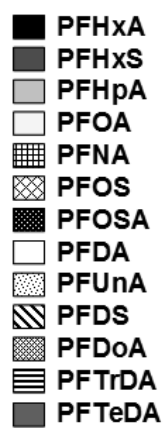

Fig. S2. Distribution patterns and total concentrations of the perfluorochemicals (PFCs), and fractions of the total occurrences of PFCs detected in sludge from the sewage treatment plants (STPs A-G; A, Stockholm; B, Gothenburg; C, Eslöv; D, Umeå; E, Borås; F, Alingsås; G, Floda) in Sweden.

\section{Abbreviations;}

PFHxA, perfluorohexane acid; PFHxS, perfluorohexane sulfonate; PFHpA, perfluoroheptane acid; PFOA, perfluorooctane acid; PFNA, perfluorononane acid; PFOS, perfluorooctane sulfonate; PFOSA, perfluorooctane sulfonamide; PFDA, perfluorodecane acid; PFUnA, perfluoroundecane acid; PFDS, perfluorodecane sulfonate; PFDoA, perfluorododecane acid; PFTrDA, perfluorotridecane acid and PFTeDA, perfluorotetradecane acid. 
Table S1. CAS numbers and chemical and physical properties of the metals, persistent organic pollutants (POPs), pharmaceuticals, and other organic contaminants (EPI Suite ${ }^{\mathrm{TM}}, 2007$ )

*priority substance included in the WFD (EU, 2000), **classified as POP according to the Stockholm Convention (UNEP, 2001), n.e., not estimated, abbreviations of the compounds used in the PCA model are presented in parenthesis

\begin{tabular}{|c|c|c|c|c|c|}
\hline Chemical & CAS RN $^{\mathrm{a}}$ & $\begin{array}{c}\mathrm{M}_{\mathrm{w}} \\
\left(\mathrm{g} \mathrm{mol}^{-1}\right)^{\mathrm{b}}\end{array}$ & $\begin{array}{l}\log { }^{c} \\
\mathrm{~K}_{\mathrm{ow}}{ }^{\mathrm{c}}\end{array}$ & $\begin{array}{c}S_{w} \\
\left(\mathrm{mg} \mathrm{L}^{-1}\right)^{d}\end{array}$ & $\begin{array}{c}\text { Henry's } \\
\text { Law } \\
\text { Constant }^{\mathrm{e}} \\
\end{array}$ \\
\hline \multicolumn{6}{|c|}{ Phenols } \\
\hline \multicolumn{6}{|l|}{ Chlorophenols (CPs) } \\
\hline 2-monochlorophenol (2-CP) & $95-57-8$ & 128.56 & 2.16 & 12734 & $4.2 \cdot 10^{-7}$ \\
\hline 3-monochlorophenol (3-CP) & $108-43-0$ & 128.56 & 2.16 & 12734 & $4.2 \cdot 10^{-7}$ \\
\hline 4-monochlorophenol (4-CP) & $106-48-9$ & 128.56 & 2.16 & 12734 & $4.2 \cdot 10^{-7}$ \\
\hline 2,3-dichlorophenol (23-CP) & $576-24-9$ & 163.00 & 2.80 & 3282 & $3.1 \cdot 10^{-7}$ \\
\hline 2,4-dichlorophenol (24-CP) & $120-83-2$ & 163.00 & 2.80 & 3282 & $3.1 \cdot 10^{-7}$ \\
\hline 2,5-dichlorophenol (25-CP) & $583-78-8$ & 163.00 & 2.80 & 3282 & $3.1 \cdot 10^{-7}$ \\
\hline 2,6-dichlorophenol (26-CP) & $87-65-0$ & 163.00 & 2.80 & 3282 & $3.1 \cdot 10^{-7}$ \\
\hline 3,4-dichlorophenol (34-CP) & $95-77-2$ & 163.00 & 2.80 & 3282 & $3.1 \cdot 10^{-7}$ \\
\hline 3,5-dichlorophenol (35-CP) & $591-35-5$ & 163.00 & 2.80 & 3282 & $3.1 \cdot 10^{-7}$ \\
\hline 2,3,4-trichlorophenol (234-CP) & $15950-66-0$ & 197.45 & 3.45 & 808 & $2.3 \cdot 10^{-7}$ \\
\hline 2,3,5-trichlorophenol (235-CP) & $933-78-8$ & 197.45 & 3.45 & 808 & $2.3 \cdot 10^{-7}$ \\
\hline 2,3,6-trichlorophenol (236-CP) & $933-75-5$ & 197.45 & 3.45 & 808 & $2.3 \cdot 10^{-7}$ \\
\hline 2,4,5-trichlorophenol (245-CP) & $95-95-4$ & 197.45 & 3.45 & 808 & $2.3 \cdot 10^{-7}$ \\
\hline 2,4,6-trichlorophenol (246-CP) & $88-06-2$ & 197.45 & 3.45 & 808 & $2.3 \cdot 10^{-7}$ \\
\hline 3,4,5-trichlorophenol (345-CP) & $609-19-8$ & 197.45 & 3.45 & 808 & $2.3 \cdot 10^{-7}$ \\
\hline 2,3,4,5-tetrachlorophenol (2345-CP) & $4901-51-3$ & 231.89 & 4.09 & 193 & $1.7 \cdot 10^{-7}$ \\
\hline 2,3,4,6-tetrachlorophenol (2346-CP) & $58-90-2$ & 231.89 & 4.09 & 193 & $1.7 \cdot 10^{-7}$ \\
\hline 2,3,5,6-tetrachlorophenol (2356-CP) & $935-95-5$ & 231.89 & 4.09 & 193 & $1.7 \cdot 10^{-7}$ \\
\hline Pentachlorophenol* (PCP) & $87-86-5$ & 266.34 & 4.74 & 45 & $1.3 \cdot 10^{-7}$ \\
\hline 4-Nonylphenol* (4NP) & $84852-15-3$ & 220.36 & 5.92 & 2.7 & $6.0 \cdot 10^{-6}$ \\
\hline Butylhydroxytoluene (BHT) & $128-37-0$ & 220.36 & 5.03 & 10 & $4.1 \cdot 10^{-6}$ \\
\hline Triclosan (TCS) & $3380-34-5$ & 289.55 & 4.66 & 9.3 & $5.0 \cdot 10^{-9}$ \\
\hline
\end{tabular}

\section{Chlorobenzenes}

\section{Polychlorobenzenes (PBCz)}

\section{1,2-dichlorobenzene (12CBz)}

1,3-dichlorobenzene (13CBz)

1,4-dichlorobenzene (14CBz)

1,2,3-trichlorobenzene* $(123 \mathrm{CBz})$

1,2,4-trichlorobenzene* (124CBz)

1,3,5-trichlorobenzene* $(135 \mathrm{CBz})$

1,2,3,4-tetrachlorobenzene (1234CBz)

1,2,3,5-tetrachlorobenzene (1235CBz)

1,2,4,5-tetrachlorobenzene (1245CBz)

Pentachlorobenzene ${ }^{* \prime * *}$ (PCBz)

Hexachlorobenzene*,** (HCBz)

$\begin{array}{rrrrl}95-50-1 & 147.00 & 3.28 & 104 & 3.0 \cdot 10^{-3} \\ 541-73-1 & 147.00 & 3.28 & 104 & 3.0 \cdot 10^{-3} \\ 106-46-7 & 147.00 & 3.28 & 104 & 3.0 \cdot 10^{-3} \\ 87-61-6 & 181.45 & 3.93 & 26 & 2.2 \cdot 10^{-3} \\ 120-82-1 & 181.45 & 3.93 & 26 & 2.2 \cdot 10^{-3} \\ 108-70-3 & 181.45 & 3.93 & 26 & 2.2 \cdot 10^{-3} \\ 634-66-2 & 215.89 & 4.57 & 6.3 & 1.6 \cdot 10^{-3} \\ 634-90-2 & 215.89 & 4.57 & 6.3 & 1.6 \cdot 10^{-3} \\ 95-94-3 & 215.89 & 4.57 & 6.3 & 1.6 \cdot 10^{-3} \\ 608-93-5 & 250.34 & 5.22 & 1.5 & 1.2 \cdot 10^{-3} \\ 118-74-1 & 284.78 & 5.86 & 0.3 & 8.9 \cdot 10^{-4}\end{array}$

Esters 
Organophosphorus compounds (OPs)

\begin{tabular}{l}
\hline Tributyl phosphate (TBP) \\
Triphenyl phosphate (TPP) \\
Tris(2-chloroethyl) phosphate (TCEP) \\
Tris(2-chloroisopropyl) phosphate (TCPP) \\
Tris(1,3-dichloro-2-propyl) phosphate \\
(TDCPP) \\
Tris(2-butoxyethyl) phosphate (TBEP) \\
Phthalates \\
\hline Dimethyl phthalate (DMP) \\
Diethyl phthalate (DEP) \\
Di- $n$-butyl phthalate (DBP) \\
Butylbenzyl phthalate (BBP) \\
Di-(2-ethylhexyl) phthalate (DEHP)* \\
Di- $n$-octyl phthalate (DNOP) \\
Di-iso-decyl phthalate (DIDP) \\
Di-iso-nonyl phthalate (DINP)
\end{tabular}

$\begin{array}{rrrrr}126-73-8 & 266.32 & 3.82 & 101 & 3.2 \cdot 10^{-6} \\ 115-86-6 & 326.29 & 4.70 & 4.7 & 4.0 \cdot 10^{-8} \\ 115-96-8 & 285.49 & 1.63 & 5597 & 2.6 \cdot 10^{-8} \\ 13674-84-5 & 327.57 & 2.89 & 740 & 6.0 \cdot 10^{-8} \\ 13674-87-8 & 430.91 & 3.65 & 30 & 2.6 \cdot 10^{-9} \\ & & & & \\ 78-51-3 & 398.48 & 3.00 & 604 & 1.2 \cdot 10^{-11} \\ & & & & \\ 131-11-3 & 194.19 & 1.66 & 7459 & 2.2 \cdot 10^{-7} \\ 84-66-2 & 222.24 & 2.65 & 720 & 3.9 \cdot 10^{-7} \\ 84-74-2 & 278.35 & 4.61 & 6.4 & 1.2 \cdot 10^{-6} \\ 85-68-7 & 312.37 & 4.84 & 1.1 & 4.2 \cdot 10^{-8} \\ 117-81-7 & 390.57 & 8.39 & 1.3 \cdot 10^{-3} & 1.2 \cdot 10^{-5} \\ 117-84-0 & 390.57 & 8.54 & 4.5 \cdot 10^{-4} & 1.2 \cdot 10^{-5} \\ 26761-40-0 & 446.68 & 10.36 & 1.0 \cdot 10^{-5} & 3.7 \cdot 10^{-5} \\ 68515-49-1 & & & & \\ 28553-12-0 & 418.62 & 9.37 & 1.2 \cdot 10^{-4} & 2.1 \cdot 10^{-5} \\ 68515-48-0 & & & & \\ & & & & \\ 1330-86-5 & 370.58 & 8.12 & 1.3 \cdot 10^{-3} & 5.2 \cdot 10^{-5} \\ 141-28-6 & 202.25 & 2.37 & 695 & 1.7 \cdot 10^{-6} \\ 141-04-8 & 258.36 & 4.19 & 18 & 5.4 \cdot 10^{-6} \\ 105-99-7 & 258.36 & 4.33 & 6.31 & 5.4 \cdot 10^{-6} \\ 103-23-1 & 370.58 & 8.12 & 1.3 \cdot 10^{-3} & 5.2 \cdot 10^{-5} \\ 123-79-5 & 370.58 & 8.26 & 4.6 \cdot 10^{-4} & 5.2 \cdot 10^{-5} \\ 105-97-5 & 426.69 & 10.23 & 3.8 \cdot 10^{-6} & 1.6 \cdot 10^{-4} \\ 133-1 & 426.69 & 10.08 & 1.1 \cdot 10^{-5} & 1.6 \cdot 10^{-4}\end{array}$

\section{Antibiotics}

\section{Fluoroquinolones (FQs)}

Norfloxacin (Nor)
Ofloxacin (Ofl)
Ciprofloxacin (Cip)

Tetracyclines (not included in the PCA model)

\begin{tabular}{l}
\hline Oxytetracycline \\
Tetracycline \\
Demeclocycline \\
Chlorotetracycline \\
Doxycycline
\end{tabular}

$\begin{array}{rrrrr}70458-96-7 & 319.34 & -0.31 & 40231 & 8.7 \cdot 10^{-19} \\ 82419-36-1 & 361.38 & -0.20 & 6873 & 5.0 \cdot 10^{-20} \\ 85721-33-1 & 331.35 & 0.28^{\mathrm{g}} & 19440 & 5.1 \cdot 10^{-19} \\ & & & & \\ & & & & \\ 2058-46-0 & 450.88 & -3.60 & 156 & 1.7 \cdot 10^{-30} \\ 64-75-5 & 482.92 & -3.70 & 3.4 \cdot 10^{5} & 1.3 \cdot 10^{-31} \\ 127-33-3 & 464.86 & -1.14 & 8.6 \cdot 10^{4} & 2.6 \cdot 10^{-24} \\ 57-62-5 & 478.89 & -0.68 & 3.8 \cdot 10^{4} & 3.5 \cdot 10^{-24} \\ 564-25-0 & 444.44 & -1.36 & 1.2 \cdot 10^{5} & 4.7 \cdot 10^{-24}\end{array}$

Pharmaceuticals (not included in the PCA model)

Non-steroid anti-inflammatory drugs

(NSAIDs)

\begin{tabular}{llllrr}
\hline Ibuprofen & $15687-27-1$ & 206.29 & 3.79 & 58 & $1.5 \cdot 10^{-7}$ \\
Naproxen & $22204-53-1$ & 230.27 & 3.10 & 44 & $3.4 \cdot 10^{-10}$ \\
Ketoprofen & $22071-15-4$ & 254.29 & 3.00 & 256 & $2.1 \cdot 10^{-11}$ \\
Diclofenac & $15307-86-5$ & 296.15 & 4.02 & 11 & $4.7 \cdot 10^{-12}$
\end{tabular}


Hormones

$\begin{array}{lrrrrr}\text { Estriol } & 50-27-1 & 288.39 & 2.81 & 558 & 1.3 \cdot 10^{-12} \\ \text { Estradiol } & 50-28-2 & 272.39 & 3.94 & 50 & 3.6 \cdot 10^{-11} \\ \text { Ethinylestradiol } & 57-63-6 & 296.41 & 4.12 & 13 & 7.9 \cdot 10^{-12} \\ \text { Norethindrone } & 68-22-4 & 298.43 & 2.99 & 30 & 5.8 \cdot 10^{-10}\end{array}$

Dioxin-like compounds

Polychlorinated biphenyls

(WHO-PCBs)**

PCB 77
PCB 81
PCB 126
PCB 169
PCB 105
PCB 114
PCB $118^{h}$
PCB 123
PCB 156
PCB 157
PCB 167
PCB 189

Polychloinated dibenzo-p-dioxins and furans (PCDD/Fs)**

2,3,7,8-TCDD (2378-CDD)
1,2,3,7,8-PeCDD (12378-CDD)
1,2,3,4,7,8-HxCDD (123478-CDD)
$1,2,3,6,7,8-H x C D D(123678-C D D)$
$1,2,3,7,8,9 . H x C D D(123789-C D D)$
$1,2,3,4,6,7,8-H p C D D(1234678-C D D)$
OCDD
$2,3,7,8-T C D F$ (2378-CDF)
$1,2,3,7,8-P e C D F(12378-C D F)$
$2,3,4,7,8-P e C D F(23478-C D F)$
$1,2,3,4,7,8-H x C D F(123478-C D F)$
$1,2,3,6,7,8-H x C D F(123678-C D F)$
$2,3,4,6,7,8-H x C D F(234678-C D F)$
$1,2,3,7,8,9-H x C D F(123789-C D F)$
$1,2,3,4,6,7,8-H p C D F(1234678-C D F)$
$1,2,3,4,7,8,9-H p C D F(1234789-C D F)$
OCDF

291.99
291.99
326.44
360.88
326.44
326.44
326.44
326.44
360.88
360.88
360.88
395.33

321.98

356.42

390.87

390.87

390.87

425.31

459.76

305.98

340.42

340.42

374.87

374.87

374.87

374.87

409.31

409.31

443.76

$\begin{array}{lll}6.34 & 3.2 \cdot 10^{-2} & 1.3 \cdot 10^{-4} \\ 6.34 & 3.2 \cdot 10^{-2} & 1.3 \cdot 10^{-4} \\ 6.98 & 7.3 \cdot 10^{-3} & 9.2 \cdot 10^{-5} \\ 7.62 & 1.6 \cdot 10^{-3} & 6.9 \cdot 10^{-5} \\ 6.98 & 7.3 \cdot 10^{-3} & 9.2 \cdot 10^{-5} \\ 6.98 & 7.3 \cdot 10^{-3} & 9.2 \cdot 10^{-5} \\ 6.98 & 7.3 \cdot 10^{-3} & 9.2 \cdot 10^{-5} \\ 6.98 & 7.3 \cdot 10^{-3} & 9.2 \cdot 10^{-5} \\ 7.62 & 1.6 \cdot 10^{-3} & 6.9 \cdot 10^{-5} \\ 7.62 & 1.6 \cdot 10^{-3} & 6.9 \cdot 10^{-5} \\ 7.62 & 1.6 \cdot 10^{-3} & 6.9 \cdot 10^{-5} \\ 8.27 & 3.7 \cdot 10^{-4} & 5.1 \cdot 10^{-5}\end{array}$

$\begin{array}{lll}6.92 & 3.8 \cdot 10^{-3} & 3.5 \cdot 10^{-6} \\ 7.56 & 8.5 \cdot 10^{-4} & 2.6 \cdot 10^{-6} \\ 8.21 & 1.9 \cdot 10^{-4} & 1.9 \cdot 10^{-6} \\ 8.21 & 1.9 \cdot 10^{-4} & 1.9 \cdot 10^{-6} \\ 8.21 & 1.9 \cdot 10^{-4} & 1.9 \cdot 10^{-6} \\ 8.85 & 4.2 \cdot 10^{-5} & 1.4 \cdot 10^{-6} \\ 9.50 & 9.3 \cdot 10^{-6} & 1.1 \cdot 10^{-6} \\ 6.29 & 3.1 \cdot 10^{-2} & 1.5 \cdot 10^{-5} \\ 6.94 & 6.9 \cdot 10^{-3} & 1.1 \cdot 10^{-5} \\ 6.94 & 6.9 \cdot 10^{-3} & 1.1 \cdot 10^{-5} \\ 7.92 & 3.5 \cdot 10^{-4} & 6.8 \cdot 10^{-6} \\ 7.92 & 3.5 \cdot 10^{-4} & 6.8 \cdot 10^{-6} \\ 7.92 & 3.5 \cdot 10^{-4} & 6.8 \cdot 10^{-6} \\ 7.58 & 1.6 \cdot 10^{-3} & 8.5 \cdot 10^{-6} \\ 8.23 & 3.5 \cdot 10^{-4} & 6.3 \cdot 10^{-6} \\ 8.23 & 3.5 \cdot 10^{-4} & 6.3 \cdot 10^{-6} \\ 8.87 & 7.6 \cdot 10^{-5} & 4.7 \cdot 10^{-6}\end{array}$


Fluorinated compounds

Perfluorochemicals (PFCs)

\begin{tabular}{l}
\hline Perfluorohexane acid (PFHxA) \\
Perfluoroheptane acid (PFHpA) \\
Perfluorooctane acid (PFOA) \\
Perfluorononane acid (PFNA) \\
Perfluorodecane acid (PFDA) \\
Perfluoroundecane acid (PFUnA) \\
Perfluorododecane acid (PFDoDA) \\
Perfluorotridecane acid (PFTrDA) \\
Perfluorotetradecane acid (PFTeDA) \\
Perfluorohexane sulfonate (PFHxS) \\
Perfluorooctane sulfonate (PFOS)** \\
Perfluorodecane sulfonate (PFDS) \\
Perfluorooctane sulfonamide (PFOSA)
\end{tabular}

Organotin compounds (OTCs)

$\begin{array}{rrrrr}307-24-4 & 314.06 & 4.37 & 0.85 & 3.3 \cdot 10^{-3} \\ 375-85-9 & 364.06 & 5.33 & 4.2 \cdot 10^{-2} & 1.7 \cdot 10^{-2} \\ 335-67-1 & 414.07 & 6.30 & 2.1 \cdot 10^{-3} & 9.1 \cdot 10^{-2} \\ 375-95-1 & 464.08 & 7.27 & 9.9 \cdot 10^{-5} & 0.48 \\ 335-76-2 & 514.09 & 8.23 & 4.7 \cdot 10^{-6} & 2.5 \\ 2058-94-8 & 564.10 & 9.20 & 5.6 \cdot 10^{-7} & 13 \\ 307-55-1 & 614.10 & 10.16 & 6.1 \cdot 10^{-7} & 69 \\ 376-03-4 & 664.11 & \text { n.e. } & \text { n.e. } & \text { n.e. } \\ 376-06-7 & 714.12 & 12.10 & 7.1 \cdot 10^{-7} & 1.9 \cdot 10^{3} \\ 3871-99-6 & 438.20 & 2.20 & 3.4 \cdot 10^{-3} & \text { n.e. } \\ 2795-39-3 & 538.22 & 4.13 & 7.6 \cdot 10^{-6} & \text { n.e. } \\ 335-77-3 & 600.14 & 8.21 & 1.1 \cdot 10^{-4} & 3.0 \cdot 10^{-1} \\ 4151-50-2 & 527.20 & 8.54 & 1.5 \cdot 10^{-4} & 5.4\end{array}$

Organometals

$\begin{array}{rrrrr}78763-54-9^{i} & 176.83 & 3.31 & 3668 & 9.7 \cdot 10^{-2} \\ 1002-53-5 & 232.94 & 0.57 & 56 & 0.38 \\ 688-73-3^{j} & 291.07 & 7.35 & 0.8 & 1.5 \\ \text { n.e. }^{j} & 196.82 & 3.05 & 2109 & 3.6 \cdot 10^{-3} \\ 1135-99-5^{\mathrm{k}} & 272.92 & 1.76 & 18 & 5.3 \cdot 10^{-4} \\ 668-34-8^{\prime} & 351.04 & 6.58 & 0.14 & 7.8 \cdot 10^{-5}\end{array}$

\section{Metals}

$\begin{array}{lrrrr}7440-38-2 & 77.95 & 0.68 & 1.4 \cdot 10^{5} & 0.77 \\ 7440-43-9 & 112.41 & -0.07 & 2.0 \cdot 10^{5} & 2.5 \cdot 10^{-2} \\ 7440-48-4 & 58.93 & 0.23 & 1.1 \cdot 10^{5} & 2.5 \cdot 10^{-2} \\ 7440-47-3 & 52.00 & 0.23 & 9.2 \cdot 10^{4} & 2.5 \cdot 10^{-2} \\ 7440-50-8 & 63.55 & -0.57 & 1.1 \cdot 10^{5} & 2.5 \cdot 10^{-2} \\ 7439-97-6 & 200.59 & 0.62 & 6.4 \cdot 10^{4} & 2.5 \cdot 10^{-2} \\ 7440-02-0 & 58.69 & -0.57 & 1.0 \cdot 10^{5} & 2.5 \cdot 10^{-2} \\ 7439-92-1 & 207.2 & 0.73 & 3.7 \cdot 10^{5} & 2.5 \cdot 10^{-2} \\ 7440-62-2 & 50.94 & 0.23 & 9.0 \cdot 10^{4} & 2.5 \cdot 10^{-2} \\ 7440-66-6 & 67.41 & -0.47 & 1.2 \cdot 10^{5} & 2.5 \cdot 10^{-2}\end{array}$

$\mathrm{As}$
$\mathrm{Cd} *$
$\mathrm{Co}$
$\mathrm{Cr}$
$\mathrm{Cu}$
$\mathrm{Hg}^{*}$
$\mathrm{Ni}^{*}$
$\mathrm{~Pb}^{*}$
$\mathrm{~V}$
$\mathrm{Zn}$

Pesticides (not included in the PCA model)

\begin{tabular}{l} 
Insecticides \\
\hline Acephate \\
$\alpha$-cypermethrine \\
Aldrin** \\
Azinophosmethyl \\
Bromopropylate \\
Cyflutrine \\
$\beta$-cyflutrine \\
Cypermethrine \\
DDD-p,p \\
DDE-p,p \\
DDT-o,p** \\
DDT-p,p** \\
Deltamethrine \\
Diazinon
\end{tabular}

$\begin{array}{rrrrr}30560-19-1 & 183.16 & -0.90 & 1.0 \cdot 10^{6} & 2.8 \cdot 10^{-12} \\ 67375-30-8 & 416.31 & 6.38 & 1.7 \cdot 10^{-2} & 7.9 \cdot 10^{-7} \\ 309-00-2 & 364.92 & 6.75 & 2.4 \cdot 10^{-3} & 3.9 \cdot 10^{-4} \\ 86-50-0 & 317.32 & 2.53 & 150 & 2.9 \cdot 10^{-10} \\ 18181-80-1 & 428.12 & 4.90 & 5.1 & 5.0 \cdot 10^{-8} \\ 68359-37-5 & 434.30 & 5.74 & 1.5 \cdot 10^{-2} & 9.2 \cdot 10^{-7} \\ 68359-37-5 & 434.30 & 5.74 & 1.5 \cdot 10^{-2} & 9.2 \cdot 10^{-7} \\ 52315-07-8 & 416.31 & 6.38 & 1.7 \cdot 10^{-2} & 7.9 \cdot 10^{-7} \\ 72-54-8 & 320.05 & 5.87 & 2.0 \cdot 10^{-2} & 4.3 \cdot 10^{-5} \\ 72-55-9 & 318.03 & 6.00 & 4.0 \cdot 10^{-2} & 3.5 \cdot 10^{-5} \\ 789-02-6 & 354.49 & 6.79 & 3.4 \cdot 10^{-3} & 1.5 \cdot 10^{-5} \\ 50-29-3 & 354.49 & 6.79 & 3.4 \cdot 10^{-3} & 1.5 \cdot 10^{-5} \\ 52918-63-5 & 505.21 & 6.18 & 4.3 \cdot 10^{-2} & 6.1 \cdot 10^{-8} \\ 333-41-5 & 304.35 & 3.86 & 110 & 8.7 \cdot 10^{-8}\end{array}$




\begin{tabular}{|c|}
\hline Dieldrin** \\
\hline Dichlorvos \\
\hline Dimethoate \\
\hline$\alpha$-endosulfan $*, * *$ \\
\hline$\beta$-endosulfan** \\
\hline Endosulfan sulfate \\
\hline Carbaryl \\
\hline Carbophenothion \\
\hline Carbofuran \\
\hline Carbosulfan \\
\hline Keto-endrin \\
\hline$\alpha$-chlordane** \\
\hline Y-chlordane** \\
\hline Chlorfenvinphos* \\
\hline Chlorobenzilate \\
\hline Chlorpyrifos* \\
\hline$\lambda$-cyhalotrin \\
\hline Methidathion \\
\hline Methoxychlor \\
\hline Parathion ethyl \\
\hline Parathion methyl \\
\hline Permethrine \\
\hline Pirimicarb \\
\hline Pirimiphos methyl \\
\hline Endrin** \\
\hline Esfenvalerate \\
\hline Fensulfothion \\
\hline Fenvalerate \\
\hline Flucythrinate \\
\hline$\alpha-\mathrm{HCH}^{*, * *}$ \\
\hline$\beta-\mathrm{HCH}^{* \prime * *}$ \\
\hline$\delta-\mathrm{HCH}^{*} * *$ \\
\hline p-HCH (Lindane)* \\
\hline Heptachlor** \\
\hline Heptachlorepoxide \\
\hline Propargite \\
\hline Propoxur \\
\hline Pyridaben \\
\hline Quinalphos \\
\hline Tetradiphone \\
\hline Herbicides \\
\hline Glyphosate \\
\hline Aminomethylphosphonic acid \\
\hline Aclonifen \\
\hline Alachlor* \\
\hline Atrazine* \\
\hline Desethylatrazine \\
\hline Desisopropylatrazine \\
\hline Cinidon ethyl \\
\hline Cyanazine \\
\hline Diflufenican \\
\hline Dichlorobenil \\
\hline Diuron* \\
\hline Isoproturon* \\
\hline Carfentrazone ethyl \\
\hline Chloridazon \\
\hline
\end{tabular}

\begin{tabular}{|c|c|c|c|c|}
\hline $60-57-1$ & 380.91 & 5.45 & $2.2 \cdot 10^{-2}$ & $5.4 \cdot 10^{-7}$ \\
\hline $62-73-7$ & 220.98 & 0.60 & $1.5 \cdot 10^{5}$ & $8.6 \cdot 10^{-7}$ \\
\hline $60-51-5$ & 229.25 & 0.28 & $1.4 \cdot 10^{5}$ & $2.1 \cdot 10^{-11}$ \\
\hline $959-98-8$ & 406.92 & 3.50 & 0.38 & $9.0 \cdot 10^{-8}$ \\
\hline $33213-65-9$ & 406.92 & 3.50 & 0.38 & $9.0 \cdot 10^{-8}$ \\
\hline $1031-07-8$ & 422.92 & 3.64 & 0.48 & $1.1 \cdot 10^{-8}$ \\
\hline $63-25-2$ & 201.23 & 2.35 & 210 & $3.1 \cdot 10^{-9}$ \\
\hline $786-19-6$ & 342.86 & 5.19 & 0.21 & $1.8 \cdot 10^{-7}$ \\
\hline $1563-66-2$ & 221.26 & 2.30 & 220 & $1.6 \cdot 10^{-9}$ \\
\hline $55285-14-8$ & 380.55 & 5.57 & 0.69 & $2.2 \cdot 10^{-9}$ \\
\hline 53494-70-5 & 380.91 & 4.99 & $7.5 \cdot 10^{-2}$ & $2.0 \cdot 10^{-8}$ \\
\hline 5103-71-9 & 409.78 & 6.26 & $3.8 \cdot 10^{-3}$ & $7.0 \cdot 10^{-5}$ \\
\hline 5566-34-7 & 409.78 & 7.00 & $1.8 \cdot 10^{-3}$ & $7.0 \cdot 10^{-5}$ \\
\hline $470-90-6$ & 359.58 & 4.15 & 20 & $5.2 \cdot 10^{-8}$ \\
\hline $510-15-6$ & 325.19 & 3.99 & 12 & $1.3 \cdot 10^{-7}$ \\
\hline $2921-88-2$ & 350.59 & 4.66 & 11 & $2.5 \cdot 10^{-6}$ \\
\hline $91465-08-6$ & 449.86 & 6.85 & $2.1 \cdot 10^{-3}$ & $1.4 \cdot 10^{-5}$ \\
\hline $950-37-8$ & 302.32 & 1.58 & $1.7 \cdot 10^{4}$ & $7.1 \cdot 10^{-9}$ \\
\hline $72-43-5$ & 345.66 & 5.67 & $1.8 \cdot 10^{-2}$ & $9.8 \cdot 10^{-8}$ \\
\hline $56-38-2$ & 291.26 & 3.73 & 8.4 & $3.0 \cdot 10^{-7}$ \\
\hline $298-00-0$ & 263.21 & 2.75 & 90 & $1.7 \cdot 10^{-7}$ \\
\hline 52645-53-1 & 391.30 & 7.43 & $2.4 \cdot 10^{-3}$ & $2.9 \cdot 10^{-7}$ \\
\hline $23103-98-2$ & 238.29 & 1.40 & $1.4 \cdot 10^{4}$ & $2.6 \cdot 10^{-9}$ \\
\hline 29232-93-7 & 305.33 & 3.44 & 240 & $2.5 \cdot 10^{-6}$ \\
\hline $72-20-8$ & 380.91 & 5.45 & $2.2 \cdot 10^{-2}$ & $5.4 \cdot 10^{-7}$ \\
\hline $66230-04-4$ & 419.91 & 6.76 & $6.2 \cdot 10^{-3}$ & $1.2 \cdot 10^{-7}$ \\
\hline $115-90-2$ & 308.35 & 2.35 & $2.0 \cdot 10^{3}$ & $1.4 \cdot 10^{-10}$ \\
\hline 51630-58-1 & 419.91 & 6.76 & $6.2 \cdot 10^{-3}$ & $1.2 \cdot 10^{-7}$ \\
\hline 70124-77-5 & 451.47 & 6.56 & $4.6 \cdot 10^{-3}$ & $3.8 \cdot 10^{-8}$ \\
\hline $319-84-6$ & 290.83 & 4.26 & 6.6 & $2.6 \cdot 10^{-4}$ \\
\hline $319-85-7$ & 290.83 & 4.26 & 6.6 & $2.6 \cdot 10^{-4}$ \\
\hline $319-86-8$ & 290.83 & 4.26 & 6.6 & $2.6 \cdot 10^{-4}$ \\
\hline $58-89-9$ & 290.83 & 4.26 & 6.6 & $2.6 \cdot 10^{-4}$ \\
\hline $76-44-8$ & 373.32 & 5.86 & $9.2 \cdot 10^{-3}$ & $1.8 \cdot 10^{-4}$ \\
\hline $1024-57-3$ & 389.32 & 4.56 & $8.4 \cdot 10^{-2}$ & $2.5 \cdot 10^{-7}$ \\
\hline $2312-35-8$ & 350.48 & 5.57 & 0.50 & $9.2 \cdot 10^{-8}$ \\
\hline $114-26-1$ & 209.25 & 1.90 & 810 & $3.4 \cdot 10^{-9}$ \\
\hline $96489-71-3$ & 364.94 & 5.47 & 0.46 & $2.3 \cdot 10^{-10}$ \\
\hline 13593-03-8 & 298.30 & 3.04 & 150 & $4.0 \cdot 10^{-9}$ \\
\hline $116-29-0$ & 356.05 & 5.18 & $2.6 \cdot 10^{-2}$ & $7.5 \cdot 10^{-8}$ \\
\hline $1071-83-6$ & 169.07 & -4.47 & $1.0 \cdot 10^{6}$ & $4.1 \cdot 10^{-19}$ \\
\hline $1066-51-9$ & 111.04 & -2.17 & $1.0 \cdot 10^{6}$ & $1.3 \cdot 10^{-15}$ \\
\hline $74070-46-5$ & 264.67 & 3.88 & 5.7 & $2.7 \cdot 10^{-9}$ \\
\hline $15972-60-8$ & 269.77 & 3.37 & 440 & $2.2 \cdot 10^{-8}$ \\
\hline $1912-24-9$ & 215.69 & 2.82 & 45 & $4.5 \cdot 10^{-9}$ \\
\hline $6190-65-4$ & 187.63 & 1.78 & 280 & $1.5 \cdot 10^{-9}$ \\
\hline $1007-28-9$ & 173.61 & 1.36 & 540 & $1.2 \cdot 10^{-9}$ \\
\hline 142891-20-1 & n.e. & n.e. & n.e. & n.e. \\
\hline $21725-46-2$ & 240.70 & 2.51 & 96 & $1.9 \cdot 10^{-12}$ \\
\hline $83164-33-4$ & 394.30 & 3.53 & 1.6 & $3.8 \cdot 10^{-12}$ \\
\hline $1194-65-6$ & 172.01 & 2.83 & 36 & $2.9 \cdot 10^{-5}$ \\
\hline $330-54-1$ & 233.10 & 2.67 & 100 & $5.3 \cdot 10^{-10}$ \\
\hline $34123-59-6$ & 206.29 & 2.84 & 92 & $1.9 \cdot 10^{-9}$ \\
\hline 128639-02-1 & 412.20 & 4.26 & 0.30 & $6.0 \cdot 10^{-11}$ \\
\hline $1698-60-8$ & 221.65 & 0.76 & $3.4 \cdot 10^{4}$ & $6.5 \cdot 10^{-12}$ \\
\hline
\end{tabular}




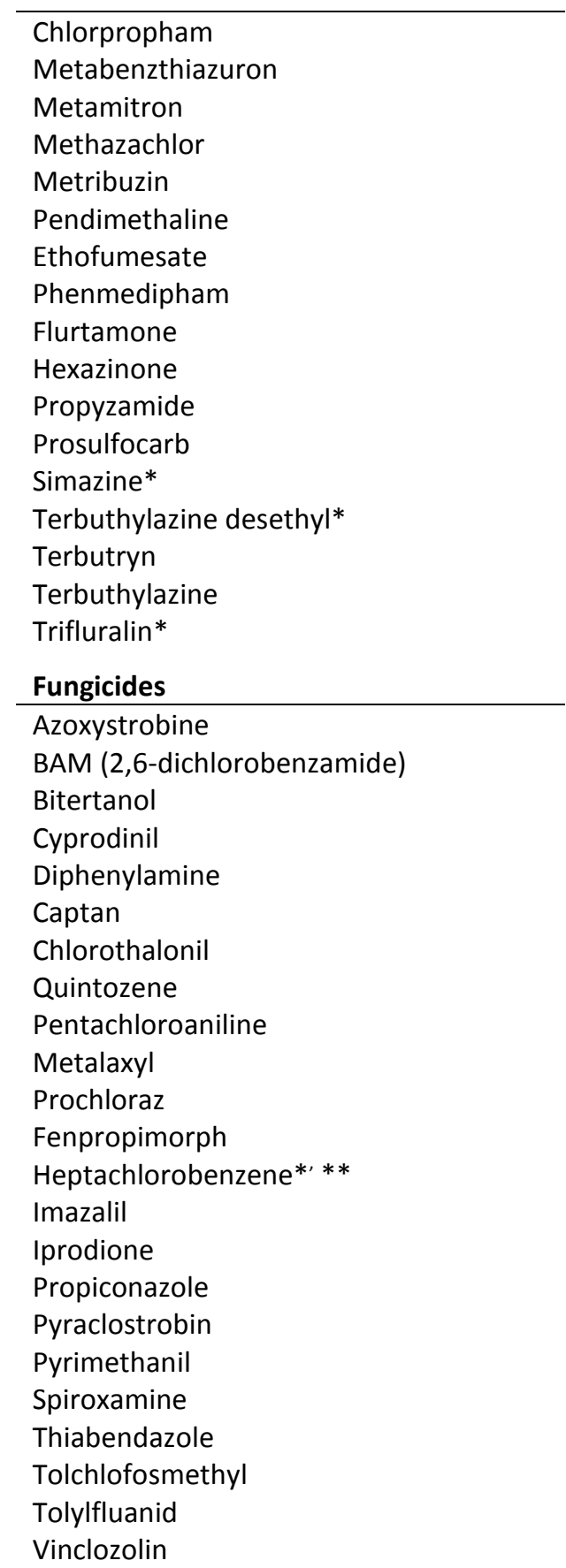

\begin{tabular}{|c|c|c|c|c|}
\hline $101-21-3$ & 213.67 & 3.30 & 49 & $2.9 \cdot 10^{-8}$ \\
\hline $18691-97-9$ & 221.28 & 2.65 & $1.1 \cdot 10^{3}$ & $6.3 \cdot 10^{-13}$ \\
\hline 41394-05-2 & 202.22 & 1.44 & $4.0 \cdot 10^{4}$ & $5.9 \cdot 10^{-12}$ \\
\hline $67129-08-2$ & 277.79 & 2.38 & 670 & $5.8 \cdot 10^{-11}$ \\
\hline 21087-64-9 & 214.29 & 1.49 & $2.1 \cdot 10^{5}$ & $1.8 \cdot 10^{-12}$ \\
\hline 40487-42-1 & 281.31 & 4.82 & 0.77 & $1.5 \cdot 10^{-6}$ \\
\hline $26225-79-6$ & 286.35 & 2.89 & 12 & $1.2 \cdot 10^{-9}$ \\
\hline 13684-63-4 & 300.32 & 3.27 & 0.68 & $5.7 \cdot 10^{-11}$ \\
\hline $96525-23-4$ & 333.31 & 2.87 & 35 & $1.7 \cdot 10^{-9}$ \\
\hline $51235-04-2$ & 252.32 & 2.15 & 590 & $4.1 \cdot 10^{-13}$ \\
\hline $23950-58-5$ & 256.13 & 3.57 & 41 & $1.0 \cdot 10^{-9}$ \\
\hline $52888-80-9$ & 251.39 & 4.23 & 5.7 & $9.4 \cdot 10^{-7}$ \\
\hline $122-34-9$ & 201.66 & 2.40 & 86 & $3.4 \cdot 10^{-9}$ \\
\hline $134-62-3$ & 191.28 & 2.26 & 170 & $2.1 \cdot 10^{-8}$ \\
\hline $886-50-0$ & 241.36 & 3.77 & 42 & $9.1 \cdot 10^{-9}$ \\
\hline $5915-41-3$ & 229.71 & 3.27 & 20 & $5.9 \cdot 10^{-9}$ \\
\hline $1582-09-8$ & 335.29 & 5.31 & $5.5 \cdot 10^{-2}$ & $2.1 \cdot 10^{-4}$ \\
\hline $131860-33-8$ & 403.40 & 1.58 & 94 & $8.0 \cdot 10^{-14}$ \\
\hline $2008-58-4$ & 190.03 & 0.90 & $1.2 \cdot 10^{3}$ & $1.2 \cdot 10^{-9}$ \\
\hline 55179-31-2 & 337.42 & 4.07 & 42 & $1.6 \cdot 10^{-12}$ \\
\hline $121552-61-2$ & 225.30 & 3.99 & 12 & $1.9 \cdot 10^{-6}$ \\
\hline $122-39-4$ & 169.23 & 3.29 & 55 & $1.1 \cdot 10^{-6}$ \\
\hline $133-06-2$ & 300.59 & 2.74 & 700 & $4.6 \cdot 10^{-9}$ \\
\hline $1897-45-6$ & 265.91 & 3.66 & 0.68 & $1.5 \cdot 10^{-7}$ \\
\hline $82-68-8$ & 295.34 & 5.03 & 0.71 & $4.8 \cdot 10^{-6}$ \\
\hline $527-20-8$ & 265.35 & 1.70 & $9.2 \cdot 10^{3}$ & $8.1 \cdot 10^{-10}$ \\
\hline 57837-19-1 & 279.34 & 4.30 & 19 & $4.3 \cdot 10^{-7}$ \\
\hline $67747-09-5$ & 376.67 & 4.13 & 3.0 & $7.6 \cdot 10^{-12}$ \\
\hline 67306-03-0 & 303.49 & 5.50 & 5.3 & $2.2 \cdot 10^{-7}$ \\
\hline $116-74-1$ & n.e. & n.e. & n.e. & n.e. \\
\hline $35554-44-0$ & 297.19 & 4.10 & 9.8 & $7.3 \cdot 10^{-8}$ \\
\hline $36734-19-7$ & 330.17 & 2.85 & $3.8 \cdot 10^{-2}$ & $1.0 \cdot 10^{-13}$ \\
\hline $60207-90-1$ & 342.23 & 4.13 & 11 & $1.4 \cdot 10^{-9}$ \\
\hline $175013-18-0$ & n.e. & n.e. & n.e. & n.e. \\
\hline $53112-28-0$ & 199.26 & 3.19 & 78 & $2.5 \cdot 10^{-6}$ \\
\hline $118134-30-8$ & 297.49 & 5.51 & 25 & $1.9 \cdot 10^{-7}$ \\
\hline $148-79-8$ & 201.25 & 2.00 & 480 & $2.0 \cdot 10^{-11}$ \\
\hline $57018-04-9$ & 301.13 & 4.77 & 3.2 & $2.6 \cdot 10^{-5}$ \\
\hline $731-27-1$ & 347.25 & 3.27 & 64 & $7.4 \cdot 10^{-7}$ \\
\hline $50471-44-8$ & 286.12 & 3.03 & 2.1 & $2.4 \cdot 10^{-6}$ \\
\hline
\end{tabular}




\begin{tabular}{|c|c|c|c|c|c|}
\hline $\begin{array}{l}\text { 2-Mercaptobenzothiazole } \\
\text { (2MERCAPTOB) }\end{array}$ & $149-30-4$ & 167.24 & 2.86 & 110 & $3.6 \cdot 10^{-8}$ \\
\hline $\begin{array}{l}\text { 2-(Tiocyanomethylthio)benzothiazole } \\
\text { (2TIOCYANOM) }\end{array}$ & $21564-17-0$ & 238.34 & 3.12 & 59 & $6.5 \cdot 10^{-12}$ \\
\hline 4-Chloro-3-cresol (KLORKRESOL) & $59-50-7$ & 142.59 & 2.70 & $4.2 \cdot 10^{3}$ & $4.6 \cdot 10^{-7}$ \\
\hline $\begin{array}{l}\mathrm{N} \text {-didecyldimethylammonium chloride } \\
\text { (DDMAC) }\end{array}$ & $7173-51-5$ & 362.09 & 4.66 & $7.2 \cdot 10^{-6}$ & $6.9 \cdot 10^{-10}$ \\
\hline Propiconazole (PROPICONA) & $60207-90-1$ & 342.23 & 4.13 & 11 & $1.4 \cdot 10^{-9}$ \\
\hline Resorcinol (RESORCINOL) & $108-46-3$ & 110.11 & 1.03 & $3.1 \cdot 10^{5}$ & $5.8 \cdot 10^{-11}$ \\
\hline Methylparabene (MEPARAB) & $99-76-3$ & 152.15 & 2.00 & $4.3 \cdot 10^{3}$ & $3.6 \cdot 10^{-9}$ \\
\hline Ethylparabene (ETPARAB) & $120-47-8$ & 166.18 & 2.49 & $1.4 \cdot 10^{3}$ & $4.8 \cdot 10^{-9}$ \\
\hline Propylparabene (PROPARAB) & $94-13-3$ & 180.21 & 2.98 & 425 & $6.4 \cdot 10^{-9}$ \\
\hline Butylparabene (BUPARAB) & $94-26-8$ & 194.23 & 3.47 & 133 & $8.5 \cdot 10^{-9}$ \\
\hline Benzylparabene (BEPARAB) & $94-18-8$ & 228.25 & 3.70 & 23 & $2.9 \cdot 10^{-10}$ \\
\hline
\end{tabular}

${ }^{a}$ CAS RN, Chemical Abstracts Service Registry Number. ${ }^{b} \mathrm{M}_{w}$, Molecular weight. ${ }^{\mathrm{c}} \log \mathrm{K}_{\mathrm{ow}}$, octanol-water partition coefficient, estimated values. ${ }^{d} S_{w}$, Water Solubility. ${ }^{e}$ atm $\cdot \mathrm{m}^{3} \mathrm{~mol}^{-1}$. ${ }^{f}$ The commercial mixture contains four isomers, of which tris(2-chloroisopropyl) phosphate is the most abundant. ${ }^{\mathrm{g}}$ experimental value (Takacs-Novak et al., 1992). ${ }^{\text {PPCB }} 118$ is classified both as WHO- and Indicator-PCB. 'Monobutyltin ion.

${ }^{\mathrm{j}}$ Tributyltin hydride. ${ }^{\mathrm{k}}$ Diphenyltin ion. 'Triphenyltin ion. 
Table S2 Comparative data of the analytical techniques and quantifying uncertainties in the analyses of the compounds

\begin{tabular}{|c|c|c|c|c|}
\hline $\begin{array}{l}\text { Class of } \\
\text { compound }\end{array}$ & Sub group & $\begin{array}{l}\text { Analytical } \\
\text { technique }^{a}\end{array}$ & $\begin{array}{l}\text { Quantifying } \\
\text { uncertainty } \\
(\%)\end{array}$ & Reference \\
\hline \multirow[t]{4}{*}{ Phenols } & Chlorophenols & GC-MS & \pm 20 & $\mathrm{CL}^{\mathrm{b}, \mathrm{c}}$ \\
\hline & Butylhydroxytoluene & GC-MS & \pm 20 & $C L^{d, e}$ \\
\hline & Triclosan & GC-MS & \pm 20 & $\mathrm{CL}^{\mathrm{d}}$ \\
\hline & 4-Nonylphenol & GC-MS & \pm 40 & $\mathrm{CL}^{\mathrm{c}}$ \\
\hline Chlorobenzenes & Polychlorobenzenes & GC-MS & \pm 30 & (Liljelind et al., 2003) $^{d}$ \\
\hline \multirow[t]{3}{*}{ Esters } & Organophosphorus compounds & GC-MS & \pm 30 & (Marklund et al., 2005) $^{d}$ \\
\hline & Phthalates & GC-MS & \pm 20 & $\mathrm{CL}^{\mathrm{d}}$ \\
\hline & Adipates & GC-MS & \pm 40 & (IVL, 2005a) \\
\hline \multirow[t]{2}{*}{ Antibiotics } & Fluoroquinolones & LC-MS/MS & \pm 20 & (Lindberg et al., 2005) $^{d}$ \\
\hline & Tetracyclines & LC-TOF-MS & \pm 40 & (IVL, 2006a) \\
\hline \multirow[t]{2}{*}{ Pharmaceuticals } & NSAIDs & GC-MS & \pm 40 & (IVL, 2006a) \\
\hline & Hormones & LC-TOF-MS & \pm 40 & (IVL, 2006a) \\
\hline \multirow{2}{*}{$\begin{array}{l}\text { Dioxin-like } \\
\text { compounds }\end{array}$} & WHO-PCBs & GC-HRMS & \pm 29 & (Liljelind et al., 2003) $^{c}$ \\
\hline & $\mathrm{PCDD} / \mathrm{Fs}$ & GC-HRMS & \pm 29 & (Liljelind et al., 2003) $^{c}$ \\
\hline \multirow[t]{6}{*}{ Other POPs } & Polycyclic aromatic hydrocarbons & GC-MS & \pm 30 & $\left(\right.$ Swedish EPA, 1990) ${ }^{c}$ \\
\hline & Indicator-PCBs & GC-MS & \pm 40 & $\left(^{(S w e d i s h ~ E P A, ~ 1990) ~}{ }^{c}\right.$ \\
\hline & Polybrominated diphenylethers & GC-MS & \pm 30 & (Liljelind et al., 2003) $^{d}$ \\
\hline & Chloroparaffins & GC-MS & \pm 30 & (Reth et al., 2005) \\
\hline & Siloxanes & ATD-GC-MS & \pm 40 & (IVL, 2005b) \\
\hline & Terpene (Limonene) & ATD-GC-FID & \pm 40 & (IVL, 2005c) \\
\hline $\begin{array}{l}\text { Fluorinated } \\
\text { compounds }\end{array}$ & Perfluorochemicals & LC-MS/MS & $\pm 5-20$ & $($ Karrman et al., 2005) \\
\hline Organometals & Organotin compounds & ICP-MS & $\pm 6-40$ & (Kumar et al., 2003) $^{d}$ \\
\hline \multirow[t]{2}{*}{ Metals } & $\mathrm{As}, \mathrm{Cd}, \mathrm{Co}, \mathrm{Cr}, \mathrm{Hg}, \mathrm{Ni}, \mathrm{Pb}$ & ICP-SFMS & \pm 20 & $\left(\right.$ U.S.EPA, 1994a) ${ }^{c}$ \\
\hline & $\mathrm{Cu}, \mathrm{V}, \mathrm{Zn}$ & ICP- AES & $\pm 20-30$ & (U.S.EPA, 1994b) ${ }^{c}$ \\
\hline Pesticides & $\begin{array}{l}\text { Insecticides, herbicides, and } \\
\text { fungicides }\end{array}$ & GC-MS & \pm 40 & $\mathrm{CL}^{\mathrm{d}}$ \\
\hline Biocides & & GC-MS & \pm 40 & (IVL, 2006b) \\
\hline
\end{tabular}

${ }^{a} \mathrm{GC}$, Gas Chromatography; MS, Mass Spectrometry; LC, Liquid Chromatography; MS/MS, Tandem Mass Spectrometry; TOF, Time-of-Flight; HRMS, High Resolution Mass Spectrometry; ATD, Automated Thermal Desorption; FID, Flame Ionization Detection; ICP, Inductively Coupled Plasma; SFMS, Sector Field Mass Spectrometry; and AES, Atomic Emission Spectrometry. ${ }^{\mathrm{b}} \mathrm{CL}$, Commercial Laboratory. ${ }^{\mathrm{C}}$ Accredited analysis. ${ }^{\mathrm{d}}$ In-house validated analytical method. ${ }^{\mathrm{e} B H T}$ semi-quantitatively analyzed, the results are presented in benzylbensoate-equivalents. ${ }^{f}$ Modification: ${ }^{13} \mathrm{C}$ labelled PCB 188 was used as recovery standard instead of $\varepsilon$ $\mathrm{HCH} .{ }^{g}$ With some modifications. 
Table S3 Concentrations of the metals, persistent organic pollutants (POPs), pharmaceuticals and personal care products (PPCPs), and other organic contaminants in sewage sludge

*priority substance included in the WFD (EU, 2000), ${ }^{* *}$ classified as POP according to the Stockholm Convention (UNEP, 2001), abbreviations of the compounds used in the PCA model are presented in parenthesis

\begin{tabular}{|c|c|c|c|c|c|c|c|}
\hline Chemical & STP A & STP B & STP C & STP D & STP E & STP F & STP G \\
\hline \multicolumn{8}{|c|}{ Phenols } \\
\hline Chlorophenols (CPs) & \multicolumn{7}{|c|}{$m g k^{-1} d . w}$. \\
\hline 2-monochlorophenol (2-CP) & 0.022 & $<0.02$ & $<0.02$ & $<0.02$ & $<0.02$ & 0.028 & 0.036 \\
\hline 3-monochlorophenol (3-CP) & $<0.02$ & $<0.02$ & $<0.02$ & $<0.02$ & $<0.02$ & $<0.02$ & $<0.02$ \\
\hline 4-monochlorophenol (4-CP) & $<0.02$ & $<0.02$ & $<0.02$ & $<0.02$ & $<0.02$ & $<0.02$ & $<0.02$ \\
\hline 2,3-dichlorophenol (23-CP) & 0.022 & $<0.02$ & $<0.02$ & $<0.02$ & $<0.02$ & 0.028 & 0.036 \\
\hline 2,4-dichlorophenol (24-CP)+ & & & & & & & \\
\hline 2,5-dichlorophenol (25-CP) & 0.042 & 0.07 & 0.034 & $<0.02$ & 0.062 & 0.021 & 0.064 \\
\hline 2,6-dichlorophenol (26-CP) & $<0.02$ & $<0.02$ & $<0.02$ & $<0.02$ & $<0.02$ & $<0.02$ & $<0.02$ \\
\hline 3,4-dichlorophenol (34-CP) & $<0.02$ & $<0.02$ & $<0.02$ & $<0.02$ & $<0.02$ & $<0.02$ & $<0.02$ \\
\hline 3,5-dichlorophenol (35-CP) & $<0.02$ & $<0.02$ & $<0.02$ & $<0.02$ & $<0.02$ & $<0.02$ & $<0.02$ \\
\hline 2,3,4-trichlorophenol (234-CP) & $<0.02$ & $<0.02$ & $<0.02$ & $<0.02$ & $<0.02$ & $<0.02$ & $<0.02$ \\
\hline 2,3,5-trichlorophenol (235-CP) & $<0.02$ & $<0.02$ & $<0.02$ & $<0.02$ & $<0.02$ & $<0.02$ & $<0.02$ \\
\hline 2,3,6-trichlorophenol (236-CP) & $<0.02$ & $<0.02$ & $<0.02$ & $<0.02$ & $<0.02$ & $<0.02$ & $<0.02$ \\
\hline 2,4,5-trichlorophenol (245-CP) & $<0.02$ & $<0.02$ & $<0.02$ & $<0.02$ & $<0.02$ & $<0.02$ & $<0.02$ \\
\hline 2,4,6-trichlorophenol (246-CP) & $<0.02$ & $<0.02$ & $<0.02$ & $<0.02$ & $<0.02$ & $<0.02$ & $<0.02$ \\
\hline 3,4,5-trichlorophenol (345-CP) & $<0.02$ & $<0.02$ & $<0.02$ & $<0.02$ & $<0.02$ & $<0.02$ & $<0.02$ \\
\hline 2,3,4,5-tetrachlorophenol (2345-CP) & $<0.02$ & $<0.02$ & $<0.02$ & $<0.02$ & $<0.02$ & $<0.02$ & $<0.02$ \\
\hline 2,3,4,6-tetrachlorophenol (2346-CP) & $<0.02$ & $<0.02$ & $<0.02$ & $<0.02$ & $<0.02$ & $<0.02$ & $<0.02$ \\
\hline 2,3,5,6-tetrachlorophenol (2356-CP) & $<0.02$ & $<0.02$ & $<0.02$ & $<0.02$ & $<0.02$ & $<0.02$ & $<0.02$ \\
\hline Pentachlorophenol* (PCP) ) & $<0.02$ & $<0.02$ & $<0.02$ & $<0.02$ & $<0.02$ & $<0.02$ & $<0.04$ \\
\hline 4-Nonylphenol* (4NP) & 20 & 21 & 8 & 12 & 40 & 13.1 & 10 \\
\hline Butylhydroxytoluene (BHT) ) & 0.81 & 0.64 & 0.83 & 1.5 & 2.4 & 1.6 & 2.2 \\
\hline Triclosan (TCS) & 4.6 & 8.3 & 1.8 & 5.5 & 3.7 & 2.4 & 3.4 \\
\hline
\end{tabular}

Chlorobenzenes

Polychlorobenzenes (PBCz)

1,2-dichlorobenzene (12CBz)

1,3-dichlorobenzene $(13 \mathrm{CBz})+$

1,4-dichlorobenzene (14CBz)

1,2,3-trichlorobenzene* $(123 \mathrm{CBz})$

1,2,4-trichlorobenzene* $(124 \mathrm{CBz})$

1,3,5-trichlorobenzene* $(135 \mathrm{CBz})$

1,2,3,4-tetrachlorobenzene (1234CBz)

1,2,3,5-tetrachlorobenzene (1235CBz) +

1,2,4,5-tetrachlorobenzene (1245CBz)

Pentachlorobenzene*,** (PCBz)

Hexachlorobenzene ${ }^{* * * *}(\mathrm{HCBz})$

$\begin{array}{ccrcccc}7 & 3 & <0.003 & <0.003 & 79 & 2 & <0.003 \\ 76 & 5 & <0.003 & 14 & 64 & 29 & 8 \\ <0.003 & <0.003 & 149 & <0.003 & <0.003 & <0.003 & <0.003 \\ <0.003 & <0.003 & 541 & <0.003 & <0.003 & <0.003 & <0.003 \\ <0.003 & <0.003 & <0.003 & <0.003 & <0.003 & <0.003 & <0.003 \\ <0.002 & <0.002 & 4 & <0.002 & <0.002 & <0.002 & <0.002 \\ 0.9 & 1 & 5 & 1 & 4 & 1 & 1 \\ 0.4 & 0.5 & 1 & 1 & 3 & 1 & 1 \\ 4 & 9 & 6 & 9 & 20 & 6 & 4\end{array}$




\section{Esters}

Organophosphorus compounds (OPs)

Tributyl phosphate (TBP)
Triphenyl phosphate (TPP)
Tris(2-chloroethyl) phosphate (TCEP)
Tris(2-chloroisopropyl) phosphate (TCPP)
Tris(1,3-dichloro-2-propyl) phosphate
(TDCPP)
Tris(2-butoxyethyl) phosphate (TBEP)
Phthalates
Dimethyl phthalate (DMP)
Diethyl phthalate (DEP)
Di- $n$-butyl phthalate (DBP)
Butylbenzyl phthalate (BBP)
Di-(2-ethylhexyl) phthalate (DEHP)*
Di- $n$-octyl phthalate (DNOP)
Di-iso-decyl phthalate (DIDP)
Di-iso-nonyl phthalate (DINP)

Adipates (not included in the PCA model)

Diethyl adipate
Di-iso-butyl adipate
Dibutyl adipate
Di-(2-ethylhexyl) adipate
Dioctyl adipate
Didecyl adipate
Di-iso-octyl adipate
Di-iso-decyl adipate

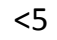

$<10$

$<10$

100

$<5$

$<10$

$<50$

$<50$

$\begin{array}{ccc}7 & 11 & 37 \\ 13 & 40 & 33 \\ 3 & 5 & 9 \\ 559 & 409 & 916 \\ 24 & 55 & 22\end{array}$

$94 \quad 210<32$

$<0.75$

$<0.70$

$<0.11$

$<0.11$

$<0.07$

$<0.70$

$<0.11$

$<0.075$

$<0.07$

$<0.11$

$<0.075$

30

32

95

$<0.07$

35

60

34

$<0.11$

14

21

$$
\mu g k g^{-1} d . w .
$$

n.m. n.m. n.m.

n.m.

n.m.

n.m.

$<5$

n.m.

n.m.

n.m.

n.m.

n.m.

$<10$

n.m.

n.m.

n.m.

n.m.

n.m. $\quad<10$

n.m.

n.m.

n.m.

n.m.

n.m.

40

n.m.

n.m.

n.m.

n.m.

n.m.

n.m.

n.m.

n.m.

n.m.

$<5$

n.m.

n.m.

n.m.

n.m.

n.m. $\quad<10$

n.m. $<50$

\section{Antibiotics}

Fluoroquinolones (FQs)

Norfloxacin (Nor)
Ofloxacin (Ofl)
Ciprofloxacin (Cip)

$m g k g^{-1}$ d.w.

$\begin{array}{llll}4.2 & 5.0 & 5.5 & 3.0 \\ 1.2 & 1.7 & 1.7 & <0.1 \\ 7.9 & 9.3 & 8.8 & 3.9 \\ {k g^{-1} \text { d.w. }} \text { d. } & & & \\ \end{array}$

Tetracyclines (not included in the PCA model)

\begin{tabular}{l}
\hline Oxytetracycline \\
Tetracycline \\
Demeclocycline \\
Chlorotetracycline \\
Doxycycline
\end{tabular}

$<4$
3000
$<4$
$<8$
1100

$\begin{array}{ccc}\text { n.m. } & 1400 & \text { n.m. } \\ \text { n.m. } & <3 & \text { n.m. } \\ \text { n.m. } & <6 & \text { n.m. } \\ \text { n.m. } & <12 & \text { n.m. } \\ \text { n.m. } & <6 & \text { n.m. }\end{array}$

n.m.

n.m.

n.m.

n.m. n.m. n.m.

n.m. n.m. n.m.

Pharmaceuticals (not included in the PCA model)

Non-steroid anti-inflammatory drugs

(NSAIDs)

Ibuprofen
Naproxen
Ketoprofen
Diclofenac

$$
\mu g k g^{-1} d . w .
$$

130
7.6
12
77

$\begin{array}{ll}\text { n.m. } & 33 \\ \text { n.m. } & <4 \\ \text { n.m. } & <9 \\ \text { n.m. } & 16\end{array}$

n.m.

n.m.

n.m. n.m.

n.m.

n.m.

n.m.

n.m.

n.m.

n.m.

n.m.

n.m.

n.m.

n.m.

n.m. 
Hormones

\begin{tabular}{|c|c|c|c|c|c|c|}
\hline Estriol & $<1$ & n.m. & $<2$ & n.m. & n.m. & n.m. \\
\hline Estradiol & $<2$ & n.m. & $<2$ & n.m. & n.m. & n.m. \\
\hline Ethinylestradiol & $<3$ & n.m. & $<4$ & n.m. & n.m. & n.m. \\
\hline Norethindrone & $<6$ & n.m. & $<9$ & n.m. & n.m. & n.m. \\
\hline
\end{tabular}

\section{Dioxin-like compounds}

Polychlorinated biphenyls (WHO-PCBs)**

\begin{tabular}{l} 
PCB 77 \\
PCB 81 \\
PCB 126 \\
PCB 169 \\
PCB 105 \\
PCB 114 \\
PCB 118 \\
PCB 123 \\
PCB 156 \\
PCB 157 \\
PCB 167 \\
PCB 189 \\
Polychloinated dibenzo-p-dioxins and -furans \\
(PCDD/Fs)** \\
\hline 2,3,7,8-TCDD (2378-CDD) \\
1,2,3,7,8-PeCDD (12378-CDD) \\
1,2,3,4,7,8-HxCDD (123478-CDD) \\
1,2,3,6,7,8-HxCDD (123678-CDD) \\
1,2,3,7,8,9.HxCDD (123789-CDD) \\
1,2,3,4,6,7,8-HpCDD (1234678-CDD) \\
OCDD \\
2,3,7,8-TCDF (2378-CDF) \\
1,2,3,7,8-PeCDF (12378-CDF) \\
2,3,4,7,8-PeCDF (23478-CDF) \\
1,2,3,4,7,8-HxCDF (123478-CDF) \\
1,2,3,6,7,8-HxCDF (123678-CDF) \\
2,3,4,6,7,8-HxCDF (234678-CDF) \\
1,2,3,7,8,9-HxCDF (123789-CDF) \\
1,2,3,4,6,7,8-HpCDF (1234678-CDF) \\
1,2,3,4,7,8,9-HpCDF (1234789-CDF) \\
OCDF
\end{tabular}

$\begin{array}{ccc}167 & 97 & 99 \\ <0.3 & <0.3 & <0.3 \\ 15 & 11 & 29 \\ 3 & 3 & 9 \\ 2314 & 1161 & 841 \\ <2 & <2 & <2 \\ 4816 & 2398 & 2009 \\ <1 & <1 & <1 \\ 1381 & 887 & 569 \\ 504 & 237 & 285 \\ <1 & <1 & <1 \\ <1 & <1 & <1\end{array}$

\section{$<0.4$}

$<1$

$<3$

8

$<3$

111

856

6

$<8<8$

$<3$

$<1$

$<1$

$<1$

$<3$

94

$<2$

491 $n g k g^{-1} d . w$.

$\begin{array}{cccc}99 & 140 & 89 & 61 \\ <0.3 & <0.3 & <0.3 & <0.3 \\ 8 & 20 & 21 & 12 \\ <0.6 & 7 & 4 & 3 \\ 1487 & 2436 & 2774 & 705 \\ <2 & <2 & <2 & <2 \\ 3227 & 4697 & 5631 & 1672 \\ <1 & <1 & <1 & <1 \\ 1152 & 1373 & 2088 & 605 \\ <1 & <1 & <1 & 140 \\ <1 & <1 & <1 & <1 \\ <1 & <1 & <1 & <1\end{array}$

$n g k g^{-1}$ d.w.

$\begin{array}{ccccccc}4 & <0.4 & <0.4 & <0.4 & <0.4 & <0.4 & <0.4 \\ & <1 & <1 & <1 & <1 & <1 & <1 \\ 5 & <3 & <3 & <3 & <3 & <3 \\ 6 & <2 & 3 & 8 & <2 & <2 \\ & <3 & <3 & <3 & <3 & <3 & <3 \\ 6 & 111 & 54 & 159 & 218 & 135 & 116 \\ & 844 & 607 & 1184 & 1224 & 1159 & 700 \\ & 7 & 4 & 3 & 8 & 5 & 4 \\ & <8 & <8 & <8 & <8 & <8 & <8 \\ & 4 & <3 & <3 & <3 & <3 & <3 \\ & <1 & <1 & <1 & <1 & <1 & <1 \\ & <1 & <1 & <1 & <1 & <1 & <1 \\ & <1 & <1 & <1 & 5 & <1 & <1 \\ & <3 & <3 & <3 & <3 & <3 & <3 \\ & 78 & 34 & 59 & 120 & 60 & 70 \\ & 3 & <2 & <2 & <2 & <2 & <2 \\ & 460 & 260 & 274 & 623 & 222 & 334\end{array}$


Other organic compounds

Polycyclic aromatic hydrocarbons (PAHs)*

(PAHsum)

\begin{tabular}{l} 
Fluoranthene \\
Benzo(b)fluoranthene \\
Benzo(k)fluoranthene \\
Benzo(a)pyrene \\
Benzo(g,h,i)perylene \\
Indeno(1,2,3-cd)pyrene \\
Polychlorinated biphenyls \\
(Indicator-PCBs)** (PCB7) \\
\hline
\end{tabular}

PCB 28

0.006

PCB 52

0.006

PCB 101

PCB $118^{\mathrm{h}}$

0.009

PCB 138

0.006

0.019

PCB 153

0.017

PCB 180

0.009

Polybrominated diphenylethers (PBDEs)

PBDE $28^{*}$
PBDE $47^{*, * *}$
PBDE $99^{* * * *}$
PBDE $100^{*, * *}$
PBDE $153^{*, * *}$
PBDE $154^{*, * *}$
PBDE $183^{* *}$
PBDE 209
Polychlorinated alkanes (PCAs)

SCCP*

0.26

$0.13 \quad 0.15$

$\mu g \mathrm{~kg}^{-1}$ d.w.

$<0.9 \quad 20 \quad 10$

0.15

0.33

0.17

0.23

31

30

37

72

$<0.9$

32

25

$<0.7<0.7$

$<0.7$

28

28

39

34

$<0.7<0.7$

$<0.7$

$<0.7$

$<0.7$

$<0.7$

$417 \quad 155$

1.4

$<0.7$

$<0.7$

$<0.7$

$<0.7$

$37 \quad 44$

508

87

354

$187 \quad 234$

$296 \quad 304$

116

55

$22 \quad 1.1$

43

190

432

237

99

MCCP

1.1

1.5

1.7

$m g \mathrm{~kg}^{-1}$ d.w.

LCCP

2.0

6.6

0.6

0.9

1.1

2.8

30

74

0.3

1.6

8.8

4.3

3.6

Siloxanes (not included in the PCA model)

Octamethylcyclotetrasiloxane

Decamethylcyclopentasiloxane

Dodecamethylcyclohexasiloxane

Hexamethyldisiloxane

Octamethyltrisiloxane

Decamethyltetrasiloxane

Dodecamethyltetrasiloxane

22

53

45

67

$\mu g \mathrm{~kg}^{-1} \mathrm{~d} . w$.

2200019000

n.m.

n.m.

310

n.m.

n.m.

$3100 \quad 2600$

n.m.

n.m.

10000

n.m.

n.m.

$<2<2$

n.m.

n.m.

2400

n.m.

n.m.

$<2 \quad<2$

n.m.

n.m.

$<2$

n.m.

n.m.

$16 \quad 13$

n.m.

n.m.

$<2$

n.m.

n.m.

$46 \quad 40$

n.m.

n.m.

n.m.

24

n.m.

n.m.

$\mu g \mathrm{~kg}^{-1} d . w$.

Terpenes (not included in the PCA model)

D-limonene

150

n.m.

n.m.

n.m.

n.m.

n.m.

82

L-limonene

350

n.m.

n.m

n.m.

n.m.

70 
Fluorinated compounds

Perfluorochemicals (PFCs)

Perfluorohexane acid (PFHxA)

Perfluoroheptane acid (PFHpA)

Perfluorooctane acid (PFOA)

Perfluorononane acid (PFNA)

Perfluorodecane acid (PFDA)

Perfluoroundecane acid (PFUnA)

Perfluorododecane acid (PFDoDA)

Perfluorotridecane acid (PFTrDA)

Perfluorotetradecane acid (PFTeDA)

Perfluorohexane sulfonate (PFHxS)

Perfluorooctane sulfonate (PFOS)**

Perfluorodecane sulfonate (PFDS)

Perfluorooctane sulfonamide (PFOSA)

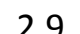

$<0.5$

$1.3 \quad 0.58$

$0.53<0.4$

$5.9<0.8$

$2.2 \quad 0.77$

$4.1<1.5$

$<1.5<1.5$

$<1.5<1.5$

$<0.3 \quad 0.36$

$67 \quad 46$

$26 \quad 5.0$

$3.5 \quad 0.88$ $\mu g \mathrm{~kg}^{-1}$ d.w.

$\begin{array}{ccccc}2.1 & 3.0 & 5.4 & <2 & <2 \\ <0.5 & 0.62 & 2.9 & <0.5 & 0.82 \\ 1.3 & 1.4 & 10 & 1.6 & 0.45 \\ <0.4 & <0.4 & 14 & 0.48 & <0.4 \\ <0.8 & <0.8 & 54 & 2.5 & 0.84 \\ <0.6 & 0.6 & 112 & 2.8 & <0.6 \\ <1.5 & <1.5 & 30 & 3.2 & 12 \\ <1.5 & <1.5 & 15 & <1.5 & <1.5 \\ <1.5 & <1.5 & 2.7 & <1.5 & <1.5 \\ 0.47 & 1.0 & 0.47 & 0.34 & <0.3 \\ 6.7 & 16 & 30 & 8.6 & 12 \\ 1.5 & 15 & 7.5 & 1.1 & 0.94 \\ 1.2 & 0.92 & 5.0 & 1.5 & 0.54\end{array}$

\section{Organometals}

Organotin compounds (OTCs)

Monobutyltin (MBT)

$228 \quad 203$

$234 \quad 243$

$9.7 \quad 16$

Tributyltin* (TBT)

Monophenyltin (MPhT)

Diphenyltin (DPhT)

Triphenyltin (TPHT)

$<0.37<0.37$

$<0.29<0.29$

$<0.43$

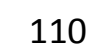

115

5.8

$<0.37$

$<0.29$

$<0.43$ $\mu g \mathrm{~kg}^{-1}$ d.w.

$\begin{array}{cccc}202 & 233 & 191 & 106 \\ 263 & 168 & 142 & 116 \\ 12 & 12 & 10 & 8.3 \\ <0.37 & <0.37 & <0.37 & <0.37 \\ <0.29 & <0.29 & <0.29 & <0.29 \\ <0.43 & <0.43 & <0.43 & <0.43\end{array}$

\section{Metals}

$\mathrm{As}$
$\mathrm{Cd}$
$\mathrm{Co}$
$\mathrm{Cr}$
$\mathrm{Cu}$
$\mathrm{Hg}^{*}$
$\mathrm{Ni}^{*}$
$\mathrm{~Pb}^{*}$
$\mathrm{~V}$
$\mathrm{Zn}$

Pesticides (not included in the PCA model)

\section{Insecticides}

Acephate
$\alpha$-cypermethrine
Aldrin**
Azinophosmethyl
Bromopropylate
Cyflutrine
$\beta$-cyflutrine
Cypermethrine
DDD-p,p
DDE-p,p

$\begin{array}{ccccccc}4.15 & 3.86 & 3.75 & 4.28 & 3.19 & 4.58 & 2.41 \\ 1.16 & 1.13 & 1.18 & 1.11 & 0.80 & 0.78 & 0.63 \\ 9.22 & 9.96 & 3.06 & 7.78 & 2.84 & 3.22 & 6.4 \\ 19.9 & 24.3 & 18.6 & 15.3 & 28.7 & 42.4 & 48.1 \\ 387 & 433 & 336 & 132 & 337 & 298 & 159 \\ 1.39 & 0.65 & 0.39 & 0.93 & 1.13 & 0.66 & 0.47 \\ 22.4 & 17.1 & 9.89 & 16.6 & 9.47 & 9.92 & 14.6 \\ 26.5 & 29.5 & 14 & 17.9 & 22 & 27.5 & 11.7 \\ 20.4 & 26.5 & 17.1 & 12.4 & 11.8 & 25.8 & 10.1 \\ 501 & 548 & 329 & 569 & 430 & 389 & 252\end{array}$

$\mu g g^{-1} d . w$.

$\begin{array}{ccccccc}\text { n.q. } & \text { n.q. } & \text { n.q. } & \text { n.q. } & \text { n.q. } & \text { n.q. } & \text { n.q. } \\ <0.006 & <0.006 & <0.006 & <0.006 & <0.006 & <0.006 & <0.006 \\ \text { n.q. } & \text { n.q. } & \text { n.q. } & \text { n.q. } & \text { n.q. } & \text { n.q. } & \text { n.q. } \\ \text { n.q. } & \text { n.q. } & \text { n.q. } & \text { n.q. } & \text { n.q. } & \text { n.q. } & \text { n.q. } \\ <0.02 & <0.02 & <0.02 & <0.02 & <0.02 & <0.02 & <0.02 \\ <0.01 & <0.01 & <0.01 & <0.01 & <0.01 & <0.01 & <0.01 \\ <0.02 & <0.02 & <0.02 & <0.02 & <0.02 & <0.02 & <0.02 \\ <0.01 & <0.01 & <0.01 & <0.01 & <0.01 & <0.01 & <0.01 \\ <0.06 & <0.01 & <0.01 & <0.05 & <0.05 & <0.05 & <0.01 \\ <0.06 & <0.01 & <0.01 & <0.01 & <0.01 & <0.03 & <0.01\end{array}$




\begin{tabular}{|c|c|c|c|c|c|c|c|}
\hline DDT-o,p** & $<0.05$ & $<0.01$ & $<0.01$ & $<0.05$ & $<0.05$ & $<0.01$ & $<0.01$ \\
\hline DDT-p,p** & $<0.05$ & $<0.02$ & $<0.02$ & $<0.05$ & $<0.05$ & $<0.06$ & $<0.02$ \\
\hline Deltamethrine & $<0.05$ & $<0.05$ & $<0.05$ & $<0.05$ & $<0.05$ & $<0.05$ & $<0.05$ \\
\hline Diazinon & $<0.03$ & $<0.03$ & $<0.03$ & $<0.03$ & $<0.03$ & $<0.03$ & $<0.03$ \\
\hline Dieldrin** & n.q. & n.q. & n.q. & n.q. & n.q. & n.q. & n.q. \\
\hline Dichlorvos & $<0.03$ & $<0.03$ & $<0.03$ & $<0.03$ & $<0.03$ & $<0.03$ & $<0.03$ \\
\hline Dimethoate & $<0.5$ & $<0.5$ & $<0.5$ & $<0.5$ & $<0.5$ & $<0.5$ & $<0.5$ \\
\hline$\alpha$-endosulfan*, ** & $<0.004$ & $<0.004$ & $<0.004$ & $<0.004$ & $<0.004$ & $<0.004$ & $<0.004$ \\
\hline$\beta$-endosulfan** & $<0.004$ & $<0.004$ & $<0.004$ & $<0.004$ & $<0.004$ & $<0.004$ & $<0.004$ \\
\hline Endosulfan sulfate & $<0.003$ & $<0.003$ & $<0.05$ & $<0.003$ & $<0.003$ & $<0.003$ & $<0.003$ \\
\hline Carbaryl & $<0.1$ & $<0.1$ & $<0.1$ & $<0.1$ & $<0.1$ & $<0.1$ & $<0.1$ \\
\hline Carbophenothion & $<0.06$ & $<0.06$ & $<0.06$ & $<0.06$ & $<0.06$ & $<0.06$ & $<0.06$ \\
\hline Carbofuran & n.q. & n.q. & n.q. & n.q. & n.q. & n.q. & n.q. \\
\hline Carbosulfan & n.q. & n.q. & n.q. & n.q. & n.q. & n.q. & n.q. \\
\hline Keto-endrin & n.q. & n.q. & n.q. & n.q. & n.q. & n.q. & n.q. \\
\hline$\alpha$-chlordane** & $<0.005$ & $<0.005$ & $<0.005$ & $<0.005$ & $<0.005$ & $<0.005$ & $<0.005$ \\
\hline$\gamma$-chlordane ${ }^{* *}$ & $<0.002$ & $<0.002$ & $<0.002$ & $<0.002$ & $<0.002$ & $<0.002$ & $<0.002$ \\
\hline Chlorfenvinphos* & $<0.004$ & $<0.004$ & $<0.004$ & $<0.004$ & $<0.004$ & $<0.004$ & $<0.004$ \\
\hline Chlorobenzilate & $<0.02$ & $<0.02$ & $<0.02$ & $<0.02$ & $<0.02$ & $<0.02$ & $<0.02$ \\
\hline Chlorpyrifos* & $<0.004$ & $<0.004$ & $<0.004$ & $<0.004$ & $<0.004$ & $<0.004$ & $<0.004$ \\
\hline$\lambda$-cyhalotrin & $<0.002$ & $<0.002$ & $<0.002$ & $<0.002$ & $<0.002$ & $<0.002$ & $<0.002$ \\
\hline Methidathion & n.q. & n.q. & n.q. & n.q. & n.q. & n.q. & n.q. \\
\hline Methoxychlor & n.q. & n.q. & n.q. & n.q. & n.q. & n.q. & n.q. \\
\hline Parathion ethyl & $<0.03$ & $<0.03$ & $<0.03$ & $<0.03$ & $<0.03$ & $<0.03$ & $<0.03$ \\
\hline Parathion methyl & n.q. & n.q. & n.q. & n.q. & n.q. & n.q. & n.q. \\
\hline Permethrine & $<0.1$ & $<0.1$ & $<0.1$ & $<0.1$ & $<0.1$ & $<0.1$ & $<0.1$ \\
\hline Pirimicarb & $<0.1$ & $<0.1$ & $<0.1$ & $<0.1$ & $<0.1$ & $<0.1$ & $<0.1$ \\
\hline Pirimiphos methyl & $<0.01$ & $<0.01$ & $<0.01$ & $<0.01$ & $<0.01$ & $<0.01$ & $<0.01$ \\
\hline Endrin** & n.q. & n.q. & n.q. & n.q. & n.q. & n.q. & n.q. \\
\hline Esfenvalerate & $<0.005$ & $<0.005$ & $<0.005$ & $<0.005$ & $<0.005$ & $<0.005$ & $<0.005$ \\
\hline Fensulfothion & $<0.1$ & $<0.1$ & $<0.1$ & $<0.1$ & $<0.1$ & $<0.1$ & $<0.1$ \\
\hline Fenvalerate & n.q. & n.q. & n.q. & n.q. & n.q. & n.q. & n.q. \\
\hline Flucythrinate & $<0.3$ & $<0.3$ & $<0.3$ & $<0.3$ & $<0.3$ & $<0.3$ & $<0.3$ \\
\hline$\alpha-\mathrm{HCH}^{*, * *}$ & $<0.004$ & $<0.004$ & $<0.004$ & $<0.004$ & $<0.004$ & $<0.004$ & $<0.004$ \\
\hline$\beta-\mathrm{HCH}^{*, * *}$ & $<0.005$ & $<0.005$ & $<0.005$ & $<0.005$ & $<0.005$ & $<0.005$ & $<0.005$ \\
\hline$\delta-\mathrm{HCH}^{*, * *}$ & $<0.005$ & $<0.005$ & $<0.005$ & $<0.005$ & $<0.005$ & $<0.005$ & $<0.005$ \\
\hline $\mathrm{\gamma}-\mathrm{HCH}$ (Lindane)* & $<0.004$ & $<0.004$ & $<0.004$ & $<0.004$ & $<0.004$ & $<0.004$ & $<0.004$ \\
\hline Heptachlor** & $<0.02$ & $<0.02$ & $<0.02$ & $<0.02$ & $<0.02$ & $<0.02$ & $<0.02$ \\
\hline Heptachlorepoxide & n.q. & n.q. & n.q. & n.q. & n.q. & n.q. & n.q. \\
\hline Propargite & n.q. & n.q. & n.q. & n.q. & n.q. & n.q. & n.q. \\
\hline Propoxur & $<0.1$ & $<0.1$ & $<0.1$ & $<0.1$ & $<0.1$ & $<0.1$ & $<0.1$ \\
\hline Pyridaben & $<0.03$ & $<0.03$ & $<0.03$ & $<0.03$ & $<0.03$ & $<0.03$ & $<0.03$ \\
\hline Quinalphos & $<0.1$ & $<0.1$ & $<0.1$ & $<0.1$ & $<0.1$ & $<0.1$ & $<0.1$ \\
\hline Tetradiphone & $<0.09$ & $<0.09$ & $<0.09$ & $<0.09$ & $<0.09$ & $<0.09$ & $<0.09$ \\
\hline Herbicides & \multicolumn{7}{|c|}{$\mu g g^{-1} d . w}$. \\
\hline Glyphosate & 0.6 & n.d. & $<2$ & 0.1 & $<2$ & n.q. & 0.7 \\
\hline Aminomethylphosphonic acid & 5 & n.d. & $<4$ & 2 & 10 & n.q. & 5 \\
\hline Aclonifen & $<0.06$ & $<0.06$ & $<0.06$ & $<0.06$ & $<0.06$ & $<0.06$ & $<0.06$ \\
\hline Alachlor* & n.q. & n.q. & n.q. & n.q. & n.q. & n.q. & n.q. \\
\hline Atrazine* & n.q. & n.q. & n.q. & n.q. & n.q. & n.q. & n.q. \\
\hline Desethylatrazine & n.q. & n.q. & n.q. & n.q. & n.q. & n.q. & n.q. \\
\hline
\end{tabular}




\begin{tabular}{|c|c|c|c|c|c|c|c|}
\hline Desisopropylatrazine & n.q. & n.q. & n.q. & n.q. & n.q. & n.q. & n.q. \\
\hline Cinidon ethyl & n.q. & n.q. & n.q. & n.q. & n.q. & n.q. & n.q. \\
\hline Cyanazine & n.q. & n.q. & n.q. & n.q. & n.q. & n.q. & n.q. \\
\hline Diflufenican & $<0.06$ & $<0.06$ & $<0.06$ & $<0.06$ & $<0.06$ & $<0.06$ & $<0.06$ \\
\hline Dichlorobenil & $<0.05$ & $<0.05$ & $<0.05$ & $<0.05$ & $<0.05$ & $<0.05$ & $<0.05$ \\
\hline Diuron* & n.q. & n.q. & n.q. & n.q. & n.q. & n.q. & n.q. \\
\hline Isoproturon* & $<0.05$ & $<0.05$ & $<0.05$ & $<0.05$ & $<0.05$ & $<0.05$ & $<0.05$ \\
\hline Carfentrazone ethyl & n.q. & n.q. & n.q. & n.q. & n.q. & n.q. & n.q. \\
\hline Chloridazon & $<0.1$ & $<0.1$ & $<0.1$ & $<0.1$ & $<0.1$ & $<0.1$ & $<0.1$ \\
\hline Chlorpropham & $<0.05$ & $<0.05$ & $<1$ & $<0.05$ & $<0.05$ & $<0.05$ & $<0.05$ \\
\hline Metabenzthiazuron & n.q. & n.q. & n.q. & n.q. & n.q. & n.q. & n.q. \\
\hline Metamitron & $<0.3$ & $<0.3$ & $<0.3$ & $<0.3$ & $<0.3$ & $<0.3$ & $<0.3$ \\
\hline Methazachlor & $<0.3$ & $<0.3$ & $<0.3$ & $<0.3$ & $<0.3$ & $<0.3$ & $<0.3$ \\
\hline Metribuzin & n.q. & n.q. & n.q. & n.q. & n.q. & n.q. & n.q. \\
\hline Pendimethaline & $<0.1$ & $<0.1$ & $<0.1$ & $<0.1$ & $<0.1$ & $<0.1$ & $<0.1$ \\
\hline Ethofumesate & $<0.05$ & $<0.05$ & $<0.05$ & $<0.05$ & $<0.05$ & $<0.05$ & $<0.05$ \\
\hline Phenmedipham & $<0.2$ & $<0.2$ & $<0.2$ & $<0.2$ & $<0.2$ & $<0.2$ & $<0.2$ \\
\hline Flurtamone & n.q. & n.q. & n.q. & n.q. & n.q. & n.q. & n.q. \\
\hline Hexazinone & $<0.03$ & $<0.03$ & $<0.03$ & $<0.03$ & $<0.03$ & $<0.03$ & $<0.03$ \\
\hline Propyzamide & $<0.06$ & $<0.06$ & $<0.06$ & $<0.06$ & $<0.06$ & $<0.06$ & $<0.06$ \\
\hline Prosulfocarb & $<0.03$ & $<0.03$ & $<0.03$ & $<0.03$ & $<0.03$ & $<0.03$ & $<0.03$ \\
\hline Simazine* & n.q. & n.q. & n.q. & n.q. & n.q. & n.q. & n.q. \\
\hline Terbuthylazine desethyl* & n.q. & n.q. & n.q. & n.q. & n.q. & n.q. & n.q. \\
\hline Terbutryn & n.q. & n.q. & n.q. & n.q. & n.q. & n.q. & n.q. \\
\hline Terbuthylazine & $<0.1$ & $<0.1$ & $<0.1$ & $<0.1$ & $<0.1$ & $<0.1$ & $<0.1$ \\
\hline Trifluralin* & $<0.005$ & $<0.005$ & $<0.005$ & $<0.005$ & $<0.005$ & $<0.005$ & $<0.005$ \\
\hline Fungicides & & & & $\mu g g^{-1} d . w$. & & & \\
\hline Azoxystrobine & n.q. & n.q. & n.q. & n.q. & n.q. & n.q. & n.q. \\
\hline BAM (2,6-dichlorobenzamide) & $<0.2$ & $<0.2$ & $<0.2$ & $<0.2$ & $<0.2$ & $<0.2$ & $<0.2$ \\
\hline Bitertanol & $<0.03$ & $<0.03$ & $<0.03$ & $<0.03$ & $<0.03$ & $<0.03$ & $<0.03$ \\
\hline Cyprodinil & $<0.2$ & $<0.2$ & $<0.2$ & $<0.2$ & $<0.2$ & $<0.2$ & $<0.2$ \\
\hline Diphenylamine & n.q. & n.q. & n.q. & n.q. & n.q. & n.q. & n.q. \\
\hline Captan & n.q. & n.q. & n.q. & n.q. & n.q. & n.q. & n.q. \\
\hline Chlorothalonil & n.q. & n.q. & n.q. & n.q. & n.q. & n.q. & n.q. \\
\hline Quintozene & n.q. & n.q. & n.q. & n.q. & n.q. & n.q. & n.q. \\
\hline Pentachloroaniline & n.q. & n.q. & n.q. & n.q. & n.q. & n.q. & n.q. \\
\hline Metalaxyl & $<0.01$ & $<0.01$ & $<0.01$ & $<0.01$ & $<0.01$ & $<0.01$ & $<0.01$ \\
\hline Prochloraz & $<0.5$ & $<0.5$ & $<0.5$ & $<0.5$ & $<0.5$ & $<0.5$ & $<0.5$ \\
\hline Fenpropimorph & n.q. & n.q. & n.q. & n.q. & n.q. & n.q. & n.q. \\
\hline Heptachlorobenzene $e^{* * *}$ & $<0.002$ & $<0.002$ & $<0.002$ & $<0.002$ & $<0.002$ & $<0.002$ & $<0.002$ \\
\hline Imazalil & $<0.2$ & $<0.2$ & $<0.2$ & $<0.2$ & $<0.2$ & $<0.2$ & $<0.2$ \\
\hline Iprodione & $<0.2$ & $<0.2$ & $<0.2$ & $<0.2$ & $<0.2$ & $<0.2$ & $<0.2$ \\
\hline Propiconazole & $<0.2$ & $<0.2$ & $<0.2$ & $<0.2$ & $<0.2$ & $<0.2$ & $<0.2$ \\
\hline Pyraclostrobin & n.q. & n.q. & n.q. & n.q. & n.q. & n.q. & n.q. \\
\hline Pyrimethanil & $<0.05$ & $<0.05$ & $<0.05$ & $<0.05$ & $<0.05$ & $<0.05$ & $<0.05$ \\
\hline Spiroxamine & n.q. & n.q. & n.q. & n.q. & n.q. & n.q. & n.q. \\
\hline Thiabendazole & $<0.3$ & $<0.3$ & $<0.3$ & $<0.3$ & $<0.3$ & $<0.3$ & $<0.3$ \\
\hline Tolchlofosmethyl & $<0.03$ & $<0.03$ & $<0.03$ & $<0.03$ & $<0.03$ & $<0.03$ & $<0.03$ \\
\hline Tolylfluanid & n.q. & n.q. & n.q. & n.q. & n.q. & n.q. & n.q. \\
\hline Vinclozolin & $<0.001$ & $<0.001$ & $<0.001$ & $<0.001$ & $<0.001$ & $<0.001$ & $<0.001$ \\
\hline
\end{tabular}




\section{Biocides}

\begin{tabular}{|c|c|c|c|c|c|c|c|}
\hline 2-Mercaptobenzothiazole (2MERCAPTOB) & $<25$ & 200 & $<20$ & 250 & 280 & 22 & 950 \\
\hline $\begin{array}{l}\text { 2-(Tiocyanomethylthio)benzothiazole } \\
\text { (2TIOCYANOM) }\end{array}$ & $<50$ & $<50$ & $<50$ & $<50$ & $<50$ & $<50$ & $<50$ \\
\hline 4-Chloro-3-cresol (KLORKRESOL) & 30 & 19 & 59 & 6.8 & 67 & 14 & $<3$ \\
\hline $\begin{array}{l}\text { N-didecyldimethylammonium chloride } \\
\text { (DDMAC) }\end{array}$ & 13000 & 14000 & 5600 & 1400 & 61000 & 21000 & 3000 \\
\hline Propiconazole (PROPICONA) & $<2$ & $<5$ & $<5$ & $<0.01$ & $<5$ & $<5$ & $<5$ \\
\hline Resorcinol (RESORCINOL) & $<10$ & $<25$ & $<50$ & $<10$ & 160 & $<20$ & $<50$ \\
\hline Methylparabene (MEPARAB) & 15 & 52 & 13 & 44 & 59 & 29 & 18 \\
\hline Ethylparabene (ETPARAB) & $<10$ & $<4$ & $<7$ & $<4$ & $<30$ & $<5$ & $<3$ \\
\hline Propylparabene (PROPARAB) & $<4$ & $<4$ & $<7$ & $<4$ & $<23$ & $<6$ & 3.6 \\
\hline Butylparabene (BUPARAB) & $<4$ & $<4$ & $<7$ & $<4$ & $<23$ & $<5$ & $<3$ \\
\hline Benzylparabene (BEPARAB) & $<6$ & $<7$ & $<12$ & $<6$ & $<36$ & $<8$ & $<5$ \\
\hline
\end{tabular}

n.m., not measured. n.q., not quantifiable. n.d., not detected.

\section{References}

CEC, 2000. Working Document on Sludge, 3rd Draft. Council of the European Community, Brussels 27 April, pp. 19.

EPI Suite ${ }^{\mathrm{TM}}$, 2007. The Estimation Program Interface (EPI) Suite. (C) 2000 U. S. Environmental Protection Agency.

EU, 1991. Council Directive 91/414/EEC of 15 July 1991 concerning the placing of plant protection products on the market. Off. J. Eur. Union L 230, 0001-0032.

EU, 2000. The EU Water Framework Directive (2000/60/EC) - integrated river basin management for Europe. European Commission.

IVL, 2005a. Results from the Swedish National Screening Programme 2004. Subreport 1: Adipates. IVL Report B1645.

IVL, 2005b. Results from the Swedish National Screening Programme 2004. Subreport 4: Siloxanes. IVL Report B1643.

IVL, 2005c. Results from the Swedish National Screening Programme 2004. Subreport 3: Limonene. IVL Report B1647.

IVL, 2006a. Results from the Swedish National Screening Programme 2005. Subreport 1: Antibiotics, Antiinflammatory substances, and Hormones. IVL. Report B1689.

IVL, 2006b. Results from the Swedish Screening 2005. Subreport 2. Biocides. IVL Report 1700.

Karrman, A., van Bavel, B., Jarnberg, U., Hardell, L., Lindstrom, G., 2005. Development of a solid-phase extraction-HPLC/single quadrupole MS method for quantification of perfluorochemicals in whole blood. Anal. Chem. 77(3), 864-870.

Kumar, S. J., Tesfalidet, S., Snell, J., Frech, W., 2003. A simple method for synthesis of organotin species to investigate extraction procedures in sediments by isotope dilution gas chromatography-inductively coupled plasma mass spectrometry - Part 1. Butyltin species. J. Anal. At. Spectrom. 18(7), 714-719.

Liljelind, P., Soderstrom, G., Hedman, B., Karlsson, S., Lundin, L., Marklund, S., 2003. Method for multiresidue determination of halogenated aromatics and PAHs in combustion-related samples. Environ. Sci. Technol. 37(16), 3680-3686.

Lindberg, R. H., Wennberg, P., Johansson, M. I., Tysklind, M., Andersson, B. A. V., 2005. Screening of human antibiotic substances and determination of weekly mass flows in five sewage treatment plants in Sweden. Environ. Sci. Technol. 39(10), 3421-3429.

Marklund, A., Andersson, B., Haglund, P., 2005. Organophosphorus flame retardants and plasticizers in Swedish sewage treatment plants. Environ. Sci. Technol. 39(19), 7423-7429.

Reth, M., Zencak, Z., Oehme, M., 2005. New quantification procedure for the analysis of chlorinated paraffins using electron capture negative ionization mass spectrometry. J. Chromatogr. A 1081(2), 225-231.

Swedish EPA, 1990. Provtagnings- och analysmetoder för slam från kommunala avloppsreningsverk (in Swedish). Swedish EPA, Rapport 3829.

Takacs-Novak, K., Jozan, M., Hermeczi, I., Szasz, G., 1992. Lipophilicity of antibacterial fluoroquinolones. Int. J. Pharm. 79, 89-96. 
U.S.EPA, 1994a. Methods for the Determination of Metals in Environmental Samples, Supplement 1 (EPA/600/R-94/111). U. S. Environmental Protection Agency, Drinking Water Methods for Chemical Contaminants (Method 200.8, modified).

U.S.EPA, 1994b. Methods for the Determination of Metals in Environmental Samples, Supplement 1 (EPA/600/R-94/111). U. S. Environmental Protection Agency, Drinking Water Methods for Chemical Contaminants (Method 200.7, modified).

UNEP, 2001. Stockholm Convention on Persistent Organic Pollutants (POPs). United Nations Environment Programme, http://www.chem.unep.ch/pops/. 